\title{
VORTEX/TOLLMIEN-SCHLICHTING WAVE INTERACTION STATES IN THE ASYMPTOTIC SUCTION BOUNDARY LAYER
}

\author{
by LIAM J. DEMPSEY ${ }^{\ddagger}$ and ANDREW G. WALTON ${ }^{\S}$ \\ (Department of Mathematics, Imperial College London, \\ South Kensington Campus, London SW7 2AZ, UK)
}

[Received April 10, 2017]

\begin{abstract}
Summary
A self-sustaining interaction between a roll/streak structure and a three-dimensional Tollmien-Schlichting wave is considered at high Reynolds number within the asymptotic suction boundary layer. Strongly nonlinear governing equations, taking the form of a vortex-wave interaction (VWI) are derived and solved numerically. Finite amplitude travelling wave states, bifurcating from the lower branch of the linear neutral curve, are obtained. These states exhibit spanwise focussing, developing steep wall-shear gradients at specific spanwise locations as the wave amplitude rises. A spanwise-local analytic analysis reveals explicitly how the solution gradually loses regularity as the nonlinearity of the VWI system is increased.
\end{abstract}

\section{Introduction}

\subsection{Preliminary Discussion}

The asymptotic suction boundary layer (ASBL) has been the focus of many theoretical, numerical and experimental studies over the years. The problem admits a simple exact Navier-Stokes solution which demonstrates clearly how the imposition of suction at the body surface prevents the growth of the boundary layer. The problem is an attractive one to study from a practical point of view because of its relevance to laminar flow control through the apparent stabilizing influence of the wall suction, while the lack of boundary layer growth referred to above renders the flow amenable to a variety of mathematical tools designed to study the stability of parallel flows.

Although there were a number of earlier analytical studies, Hughes and Reid (1) were the first to show that it is possible to derive an exact analogue of the Orr-Sommerfeld equation governing the finite Reynolds number stability of the flow, with the Reynolds number Re defined in terms of the ratio of the freestream velocity to the wall suction. Hocking (2), as part of his weakly nonlinear analysis of ASBL, was the first to accurately solve this equation, obtaining a value $\mathrm{Re} \simeq 54370$ for the linear critical Reynolds number for instability (or equivalently a critical value of the wall suction which stabilises the flow to small disturbances). Later work by Fransson and Alfredsson (3) confirmed this value, while also investigating the flow properties experimentally. They found that, although the

‡ (ljdempsey@hotmail.co.uk)

$\S$ Corresponding author (a.walton@imperial.ac.uk)

Q. Jl Mech. Appl. Math. (2016) ?? (??), 1-28

(C) Oxford University Press 2016 
general trend of the stabilizing effect of suction predicted by linear theory held true, the flow exhibited instability at values of Re considerably less than the linear critical value. The observation that nonlinear effects are particularly relevant in the transition process for this flow was consistent with an earlier numerical investigation by Milinazzo and Saffman (4). They tracked two-dimensional finite amplitude states bifurcating from the linear neutral curve down to $\operatorname{Re} \simeq 3000$. Although in itself this fall in the critical value of Re due to nonlinearity is quite remarkable, subsequent studies have found that the inclusion of three-dimensional effects alongside nonlinearity can reduce this value by a further order of magnitude, down to $\mathrm{Re} \simeq 350$. Using techniques developed for tracking nonlinear solutions in plane Couette flow, Khapko et al. (5) and Kreilos et al. (6) have recently identified steady three-dimensional time-periodic states, while Deguchi and Hall (7) and Kreilos et al. (8) have discovered travelling-wave solutions.

These latter solutions are interesting as they exhibit a spanwise localisation which is thought to be the precursor to the formation of turbulent patches in the flow-field. The solutions also acquire a clear multi-layer asymptotic structure as Re increases and they can be visualized as an interaction between three distinct structures: a flow in the crossstream plane, independent of downstream location, known as a roll, a streamwise component possessing some cross-stream variation (a streak) and a three-dimensional travelling wave propagating in the streamwise direction. This roll/streak/wave interaction is often referred to at finite Re as a coherent structure or self-sustained process (SSP) (9) and as a vortexwave interaction (VWI) (10) at asymptotically large Re.

The travelling wave states discovered in $(\mathbf{7})$ involve waves that are either propagating close to the freestream speed or are governed primarily by inviscid dynamics. In both cases the families of solutions were found by employing a homotopy strategy from corresponding solutions in plane Couette flow: they do not appear to have any connection to the linear neutral curve for ASBL. In the work presented here we consider a self-sustaining process which does have a direct connection to this curve. We will construct an asymptotic framework based on the vortex/Tollmien-Schlichting (TS) wave interaction formulated for the Blasius boundary layer by Hall and Smith (11). This allows us to describe a roll/streak/wave interaction in ASBL at high Re, which involves three-dimensional waves possessing viscous TS characteristics. When we solve the governing interaction equations in section 5 we are in essence carrying out a three-dimensional version of the calculation in (4), identifying finite-amplitude states that bifurcate from the ASBL linear neutral curve at high Re. Although these solutions possess a very different asymptotic structure to those in (7), there are some common properties, such as the development of steep local spanwise gradients with increasing nonlinearity.

\section{$1.2 \quad$ Non-dimensionalisation and governing equations}

In what follows we will use a hatted variable to denote a dimensional quantity. Consider the flow of a viscous incompressible fluid, with density $\widehat{\rho}$ and kinematic viscosity $\widehat{\nu}$, over an infinite flat plate positioned at $\widehat{y}=0$ with constant wall suction $-\widehat{V}_{0}$ and freestream streamwise velocity $\widehat{U}_{0}$, where $\widehat{U}_{0}$ and $\widehat{V}_{0}$ are positive. In this scenario $(\widehat{x}, \widehat{y}, \widehat{z})$ are, respectively, the streamwise, wall-normal and spanwise directions with corresponding velocities $\widehat{\mathbf{u}}=(\widehat{u}, \widehat{v}, \widehat{w})$. This flow is governed by the unsteady incompressible Navier-Stokes 
equations

$$
\widehat{\nabla} \cdot \widehat{\mathbf{u}}=\mathbf{0}, \quad \widehat{\mathbf{u}}_{\widehat{t}}+(\widehat{\mathbf{u}} \cdot \widehat{\nabla}) \widehat{\mathbf{u}}=-\frac{1}{\widehat{\rho}} \widehat{\nabla} \widehat{p}+\widehat{\nu} \widehat{\nabla}^{2} \widehat{\mathbf{u}},
$$

with no slip boundary conditions on the plate

$$
\widehat{\mathbf{u}}=\left(0,-\widehat{V}_{0}, 0\right) \text { on } \widehat{y}=0,
$$

and far field matching conditions with the freestream velocity,

$$
\widehat{\mathbf{u}} \rightarrow\left(\widehat{U}_{0},-\widehat{V}_{0}, 0\right) \text { as } \widehat{y} \rightarrow \infty .
$$

The simple well-known solution to these equations is

$$
\widehat{u}=\widehat{U}_{0}\left\{1-\exp \left(-\widehat{V}_{0} \widehat{y} / \widehat{\nu}\right)\right\}, \widehat{v}=-\widehat{V}_{0}, \widehat{w}=0, \widehat{p}=\widehat{p}_{0},
$$

where $\widehat{p}_{0}$ is a constant. This solution identifies characteristic scales motivating the nondimensionalisation of the Navier-Stokes equations using the velocity scale $\widehat{U}_{0}$, length scale $\widehat{\nu} / \widehat{V}_{0}$, time scale $\widehat{\nu} /\left(\widehat{U}_{0} \widehat{V}_{0}\right)$ and pressure scale $\widehat{\rho} \widehat{U}_{0}^{2}$. With these scales it is natural to define the Reynolds number as

$$
\operatorname{Re} \equiv \widehat{U}_{0} / \widehat{V}_{0}
$$

and the Navier-Stokes equations can be written

$$
\begin{aligned}
\nabla \cdot \mathbf{u} & =\mathbf{0} \\
\mathbf{u}_{t}+(\mathbf{u} \cdot \nabla) \mathbf{u} & =-\nabla p+\operatorname{Re}^{-1} \nabla^{2} \mathbf{u}
\end{aligned}
$$

with corresponding dimensionless boundary conditions

$$
\mathbf{u}=\left(0,-\operatorname{Re}^{-1}, 0\right) \text { on } y=0, \mathbf{u} \rightarrow\left(1,-\operatorname{Re}^{-1}, 0\right) \text { as } y \rightarrow \infty .
$$

The basic state written in terms of non-dimensional coordinates is

$$
\mathbf{u}=\mathbf{u}_{b}=\left(u_{b}, \operatorname{Re}^{-1} v_{b}, 0\right), \quad p=p_{0},
$$

where

$$
u_{b}=1-\mathrm{e}^{-y}, \quad v_{b}=-1 .
$$

The primary objective of this paper is to find alternative solutions to the system (1.6), (1.7) aside from the simple two-dimensional basic state (1.8), (1.9). We will do this by tracking three-dimensional travelling wave equilibrium solutions which bifurcate from the basic state via a viscous linear instability. This could be carried out at finite Re by seeking travelling wave equilibrium states of the full Navier-Stokes equations and assuming various symmetries, but here instead we will consider the problem of computing these states at asymptotically large Re where we can identify the leading order dynamics and gain an understanding of how the state is self sustained. The states that we will track lie, for small amplitude, on the linear neutral curve for three-dimensional disturbances. In the next section we show how this curve can be determined for all Reynolds numbers before going on to specifically consider the lower branch at high Re. Following this, the remainder of the paper is as follows. In section 3 we motivate the scalings and derive the governing interaction 
equations for our VWI in ASBL. A weakly nonlinear analysis of these equations follows in section 4 where we identify precisely how the solution bifurcates from the lower branch of the linear neutral curve found in section 2. This small amplitude solution also serves as a primal seed for the computation of the full numerical problem which is studied in section 5, where, among other things, the solution is found to develop steep spanwise gradients at specific spanwise locations as the wave amplitude is increased. The loss of regularity of the solution, which accompanies this steepening phenomenon, is studied theoretically in section 6 , with some conclusions and avenues for further research presented in section 7 .

\section{Linear stability of the asymptotic suction boundary layer}

\subsection{Stability at finite Reynolds number}

Assuming small perturbations to the basic state we write

$$
(\mathbf{u}, p)=\left(u_{b}(y),-\operatorname{Re}^{-1}, 0, p_{0}\right)+\Delta(\widetilde{u}(y), \widetilde{v}(y), \widetilde{w}(y), \widetilde{p}(y)) E+c . c ., \quad E \equiv \mathrm{e}^{\mathrm{i} \bar{\alpha}(x-\bar{c} t)+\mathrm{i} \bar{\beta} z},
$$

where c.c. denotes complex conjugate. After substitution into the Navier-Stokes equations (1.6) and linearisation, we obtain the perturbation equations

$$
\begin{aligned}
\mathrm{i} \bar{\alpha} \widetilde{u}+\widetilde{v}^{\prime}+\mathrm{i} \bar{\beta} \widetilde{w} & =0 \\
\mathrm{i} \bar{\alpha}\left(u_{b}-\bar{c}\right) \widetilde{u}+\widetilde{v} u_{b}^{\prime} & =-\mathrm{i} \bar{\alpha} \widetilde{p}+\operatorname{Re}^{-1}\left(\widetilde{u}^{\prime \prime}-\bar{\alpha}^{2} \widetilde{u}\right), \\
\mathrm{i} \bar{\alpha}\left(u_{b}-\bar{c}\right) \widetilde{v} & =-\widetilde{p}^{\prime}+\operatorname{Re}^{-1}\left(\widetilde{v}^{\prime \prime}-\bar{\alpha}^{2} \widetilde{v}\right) \\
\mathrm{i} \bar{\alpha}\left(u_{b}-\bar{c}\right) \widetilde{w} & =-\mathrm{i} \bar{\beta} \widetilde{p}+\operatorname{Re}^{-1}\left(\widetilde{w}^{\prime \prime}-\bar{\alpha}^{2} \widetilde{w}\right)
\end{aligned}
$$

If we then eliminate the pressure, we find that the normal velocity perturbation satisfies the following modified Orr-Sommerfeld equation:

$$
\widetilde{v}^{\prime \prime \prime \prime}-2 \bar{\chi}^{2} \widetilde{v}^{\prime \prime}+\bar{\chi}^{4} \widetilde{v}+\left[\widetilde{v}^{\prime \prime \prime}-\bar{\chi}^{2} \widetilde{v}^{\prime}\right]=(\mathrm{i} \bar{\alpha} \operatorname{Re})\left\{\left(u_{b}-\bar{c}\right)\left(\widetilde{v}^{\prime \prime}-\bar{\chi}^{2} \widetilde{v}\right)-u_{b}^{\prime \prime} \widetilde{v}\right\},
$$

where $\bar{\chi}^{2}=\bar{\alpha}^{2}+\bar{\beta}^{2}$, as first derived in (1). Here the two terms on the left-hand-side in square brackets represent the effects of suction and are absent from the traditional OrrSommerfeld equation for parallel flows. It is also worth remarking that, aside from the linearisation process, equation (2.3) is exact, with no heuristic parallel flow approximations necessary, as is the case for the Blasius boundary layer, for example. The usual no-slip boundary conditions are to be applied at the wall, together with decay of the perturbations at infinity:

$$
\widetilde{v}=\widetilde{v}^{\prime}=0 \text { on } y=0 \text { and as } y \rightarrow \infty .
$$

The eigenvalue problem consisting of (2.3), (2.4) can be solved easily by a collocation method involving the use of Chebyshev polynomials in the wall normal direction. The semi-infinite domain is dealt with by considering (1.8), (1.9) as the limit as $h \rightarrow \infty$ of the corresponding asymptotic suction boundary layer with a 'finite lid' of height $h$. This has the basic solution

$$
u_{b, h}=\left(1-\mathrm{e}^{-y}\right) /\left(1-\mathrm{e}^{-h}\right), \quad(0<y<h),
$$

with $v=v_{b}=-\operatorname{Re}^{-1}$ as in the semi-infinite problem and with the outer boundary conditions given in (2.4) now applied on $y=h$. It transpires that as $h$ is increased, the neutral stability 

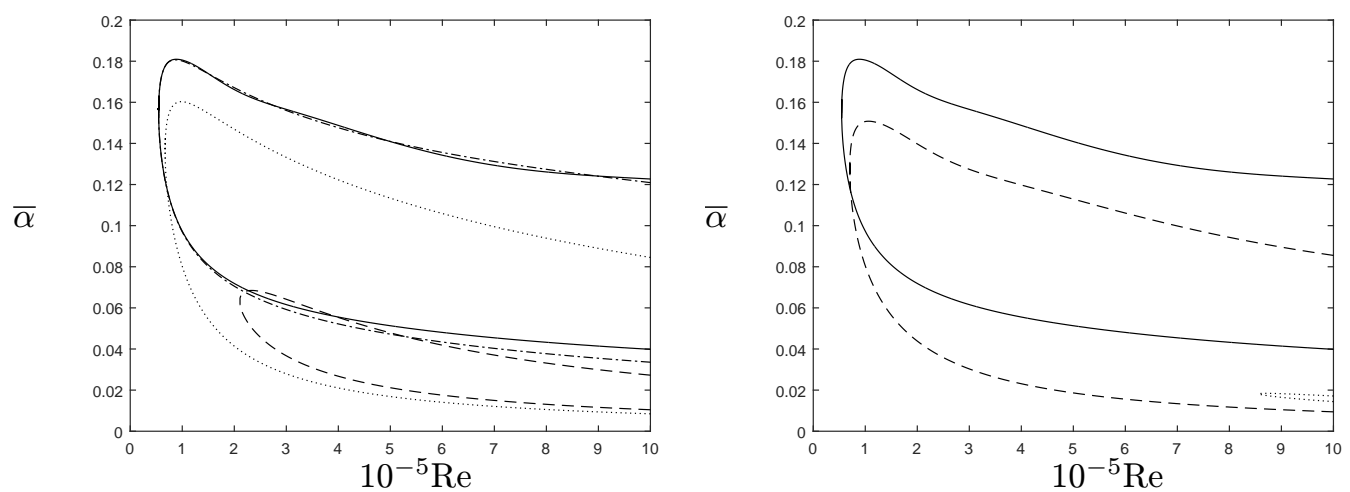

Fig. 1 (a) Neutral curves for the 'finite lid' ASBL from the numerical solution of (2.3) over the domain $0<y<h$. The curves shown are for spanwise wavenumber $\bar{\beta}=0$ and various values of the parameter $h: h=7$ (dashed), $h=10$ (dotted), $h=30$ (dashdot), $h=50$ (solid). (b) Neutral curves for 'infinite' ASBL from the numerical solution of (2.3) over the domain $0<y<\infty$ for various spanwise wavenumbers $\bar{\beta}: \bar{\beta}=0$ (solid), $\bar{\beta}=0.1$ (dashed), $\bar{\beta}=0.18$ (dotted).

curve (along which the imaginary part of $\bar{c}$ is zero) converges fairly rapidly to the $h=\infty$ case and in practice it is sufficient to take $h=50$ in order to compute an accurate curve for the semi-infinite situation. The convergence is demonstrated in Fig. 1a where we show neutral curves for spanwise wavenumber $\bar{\beta}=0$ at various values of $h$. Neutral curves for the semiinfinite problem are shown in Fig. $1 \mathrm{~b}$ for various values of $\bar{\beta}$. One can see that in fact the $2 \mathrm{D}$ mode is most unstable with a critical Reynolds number $\mathrm{Re} \simeq 54370$ which as mentioned earlier was first derived accurately in (2). The fact that $\bar{\beta}=0$ is the most unstable case is of course simply a consequence of the fact that the linear stability equations can be recast in quasi-two-dimensional form by the use of Squire's theorem. The identification of the $2 \mathrm{D}$ mode as most unstable is in fact misleading, since recent numerical simulations have shown that once nonlinearity is taken into account, the states with the lowest critical Re are inherently three-dimensional. Indeed, 3D waves are a crucial ingredient of the self-sustaining process we consider in section 3 .

\subsection{Asymptotically large Reynolds number: lower branch}

It can be seen that the neutral curves computed for the semi-infinite case possess two distinct branches along which the wavenumber tends to zero as the Reynolds number tends to infinity. Here we will concentrate on the lower branch at large Re where the so-called critical layer (where $u_{b}=\bar{c}$ ) is situated within a thin viscous wall layer. The first step is to deduce the asymptotic properties of the lower branch linear neutral modes. Once this is known we can increase the amplitude of the wave until nonlinear effects come into play and the interaction falls within the strongly nonlinear regime. The structure for the interaction can be seen to follow from that described in (11) for the Blasius boundary layer, but for completeness we will provide a derivation of the key scales and equations here.

At large Reynolds numbers these lower branch solutions possess a triple deck structure. We will now motivate the scales for these modes. Since we are only considering linear 
effects at present we can take our governing equations to be the perturbation system (2.2). Alternatively we could directly analyze the modified Orr-Sommerfeld equation (2.3) as $\operatorname{Re} \rightarrow \infty$ : however the system (2.2) is more straightforward to analyse and, importantly, allows us to generalize our arguments to the nonlinear case (section 3). In the arguments that follow we will use subscripts $L, M$ and $U$ to denote the perturbations to the basic state in the lower, main and upper decks respectively. We assume the disturbances possess a streamwise wavenumber $\bar{\alpha}$, a spanwise wavenumber $\bar{\beta}$ and a wavespeed $\bar{c}$, all of which are real. For convenience, in the discussion which follows we let $\varepsilon$ denote the size of the streamwise wavenumber $\bar{\alpha}$. One of our aims is to determine $\varepsilon$ in terms of Re.

Let us start by considering the main deck: this represents the main part of the flow-field in which the unperturbed flow is given by (1.8), (1.9) with $y$ of $O(1)$ and we assume the phasespeed $\bar{c} \ll 1$. If we now turn to the sizes of the perturbations and take $\widetilde{u}_{M} \sim O(1)$ without loss of generality, the continuity equation (2.2a) implies that $\widetilde{v}_{M} \sim \varepsilon$, while the linearized spanwise momentum equation $(2.2 \mathrm{~d})$ yields that $\widetilde{w}_{M} \sim \bar{\beta}_{\bar{p}} / \varepsilon$. It turns out that the dominant balance in the wall normal momentum equation $(2.2 \mathrm{c})$ is just $\widetilde{p}^{\prime}=0$, and thus the pressure is a constant throughout the main deck. By matching the pressure with the upper and lower decks we conclude that the pressure is of the same order throughout the whole structure, i.e. $\widetilde{p}_{L} \sim \widetilde{p}_{M} \sim \widetilde{p}_{U}$. We will find when we set out the governing equations in the main deck that the streamwise velocity perturbation $\widetilde{u}_{M}$ tends to a constant as $y \rightarrow 0$, leading to a slip velocity and hence necessitating the existence of a viscous sublayer (the lower deck) in order to satisfy the no-slip condition on the wall: thus $\widetilde{u}_{L}$ is $O(1)$. In addition it turns out that the normal velocity perturbation $\widetilde{v}_{M}$ tends to a constant as $y \rightarrow \infty$ and hence we have $v_{U}=O(\varepsilon)$.

Now we consider the upper deck whose thickness, $y_{U}$ say, is to be determined. In this region the non-dimensional basic state velocity is $(1,0,0)$ at leading order, and balancing of terms in the linearized momentum equations $(2.2 \mathrm{~b})-(2.2 \mathrm{~d})$ reveals that $\widetilde{p} \sim \widetilde{u}_{U}, \varepsilon \widetilde{v}_{U} y_{U}, \varepsilon \widetilde{w}_{U} / \bar{\beta}$ respectively, while from the continuity equation (2.2a) we find $\varepsilon \widetilde{u}_{U} \sim \widetilde{v}_{U} / y_{U} \sim \bar{\beta} \widetilde{w}_{U}$. These balances identify the lower branch spanwise wavenumber scaling as $\bar{\beta} \sim \varepsilon$ and the thickness of the upper deck as $y_{U} \sim \varepsilon^{-1}$. It also follows that $\widetilde{u}_{U}, \widetilde{v}_{U}, \widetilde{w}_{U}$ are all of $O(\varepsilon)$ in this layer, and the pressure perturbation throughout the whole structure is $\widetilde{p} \sim O(\varepsilon)$.

Finally, in order to derive the wavenumber and phasespeed scalings we need to consider the lower deck. A Taylor expansion of the basic state (1.9) for small $y$ indicates that $u_{b} \sim y_{L}$ and we already know from above that $\widetilde{u}_{L}=O(1)$. The continuity equation (2.2a) then reveals the balances $\varepsilon \sim \widetilde{v}_{L} / y_{L} \sim \bar{\beta} \widetilde{w}_{L}$ from which we conclude (in view of the scaling for $\bar{\beta}$ ) that $\widetilde{w}_{L}$ is $O(1)$ and $v_{L} \sim \varepsilon y_{L}$. Finally by demanding that there is an inertialviscous-pressure-gradient balance in this layer, equation $(2.2 \mathrm{~b})$ yields that $\varepsilon \bar{c} \widetilde{u}_{L} \sim \varepsilon y_{L} \widetilde{u}_{L} \sim$ $\varepsilon \widetilde{p} \sim \operatorname{Re}^{-1} \widetilde{u}_{L} / y_{L}^{2}$ from which we can deduce that $y_{L}=O(\varepsilon)$ and

$$
\varepsilon=\operatorname{Re}^{-1 / 4}
$$

is determined uniquely. It can also be seen that the phasespeed $\bar{c}=O(\varepsilon)$. For convenience we now define the relevant streamwise, spanwise and temporal scales for the lower branch Tollmien-Schlichting wave which consists of the slow variables

$$
(X, Z)=\varepsilon(x, z), \quad T=\varepsilon^{2} t .
$$


In terms of these variables the travelling wave component can be conveniently represented as

$$
E=\exp \{\mathrm{i} \alpha(X-c T)+\mathrm{i} \beta Z\} .
$$

with $\alpha, c, \beta$ all $O(1)$ quantities. It follows that the relationship between the wavenumbers and phasespeed of the finite Re perturbation and the corresponding asymptotically scaled quantities is

$$
(\bar{\alpha}, \bar{\beta}, \bar{c})=\operatorname{Re}^{-1 / 4}(\alpha, \beta, c) .
$$

In fact the scalings derived here can be deduced from the more familiar ones for Blasius flow at Reynolds number $\mathrm{R}_{b}$ over a plate of length $\widehat{L}$ as follows. Writing the dimensional boundary-layer thickness as $\widehat{\delta}$, we then have $\widehat{\delta}=\widehat{\nu} / \widehat{V}_{0}=\mathrm{R}_{b}^{-1 / 2} \widehat{L}$, and hence $\mathrm{Re}=\mathrm{R}_{b}^{1 / 2}$. Thus, for example, the dimensional thickness of the lower deck is $\mathrm{Re}^{-1 / 4} \widehat{\delta}=\mathrm{R}_{b}^{-5 / 8} \widehat{L}$, which is the Blasius scaling first derived in (12), and assumed in (11).

Now that we have completed our order of magnitude scaling argument we will set out formally the expansions discussed above in each deck. In order to facilitate the generalization to nonlinear interactions we will now take as the governing equations the dimensionless Navier-Stokes equations (1.6), rather than the linearized version (2.2) we have used thus far. All the scalings set out below are deduced from the order of magnitude analysis we have just presented.

\subsubsection{The main deck expansions}

Within the main deck, where $y \sim O(1)$, the flow variables expand as

$$
\begin{aligned}
u & =u_{b}+\sigma\left[u_{11}(y) E+c . c .\right]+\cdots \\
v & =\varepsilon^{4} v_{b}+\sigma \varepsilon\left[v_{11}(y) E+c . c .\right]+\cdots, \\
w & =\sigma \varepsilon\left[w_{11}(y) E+c . c .\right]+\cdots \\
p & =p_{0}+\sigma \varepsilon\left[p_{11} E+\text { c.c. }\right]+\cdots,
\end{aligned}
$$

where $\sigma$ is a sufficiently small parameter that all nonlinear terms can be ignored (later in section 3 we will determine the critical size for $\sigma$ such that nonlinearity enters the lower deck). Substituting (2.9) into (1.6), we find that the governing equations for the perturbations in the main deck are

$$
\mathrm{i} \alpha u_{11}+v_{11}^{\prime}=0, \quad \mathrm{i} \alpha u_{b} u_{11}+u_{b}^{\prime} v_{11}=0, \quad \mathrm{i} \alpha u_{b} w_{11}+\mathrm{i} \beta p_{11}=0,
$$

where $p_{11}$ is a constant. It is worth mentioning here that equations (2.10) are quasi-steady because the phasespeed is small (specifically of $O(\varepsilon)$ ). These equations admit the solution

$$
u_{11}=A u_{b}^{\prime}(y), \quad v_{11}=-i \alpha A u_{b}(y), \quad w_{11}=-\frac{\beta}{\alpha u_{b}(y)} p_{11},
$$

where $A$ is an arbitrary constant. We see that $u_{11}=A$ on $y=0$, and $v_{11} \rightarrow-\mathrm{i} \alpha A$ as $y \rightarrow \infty$, results alluded to earlier when we carried out our scaling arguments. 


\subsubsection{The upper deck expansions}

Motivated by our scaling analysis we introduce the upper deck wall normal variable $\bar{Y}=\varepsilon y$ and the flow expansion

$$
\begin{aligned}
u & =1+\sigma \varepsilon\left[\tilde{u}_{1}(\bar{Y}) E+c . c .\right]+\cdots \\
v & =\varepsilon^{4} v_{b}+\sigma \varepsilon\left[\tilde{v}_{1}(\bar{Y}) E+c . c .\right]+\cdots \\
w & =\sigma \varepsilon\left[\tilde{w}_{1}(\bar{Y}) E+c . c .\right]+\cdots \\
p & =p_{0}+\sigma \varepsilon\left[\tilde{p}_{1}(\bar{Y}) E+c . c .\right]+\cdots
\end{aligned}
$$

Substituting (2.12) into (1.6) we obtain the inviscid balances

$$
\begin{aligned}
\mathrm{i} \alpha \tilde{u}_{1}+\tilde{v}_{1}^{\prime}+\mathrm{i} \beta \tilde{p}_{1} & =0 \\
\mathrm{i} \alpha \tilde{u}_{1}+\mathrm{i} \alpha \tilde{p}_{1} & =0 \\
\mathrm{i} \alpha \tilde{v}_{1}+\tilde{p}_{1}^{\prime} & =0 \\
\mathrm{i} \alpha \tilde{w}_{1}+\mathrm{i} \beta \tilde{p}_{1} & =0 .
\end{aligned}
$$

Combining these equations to eliminate $\tilde{u}_{1}, \tilde{v}_{1}$ and $\tilde{w}_{1}$, we are left with a single equation for the upper deck pressure $\tilde{p}_{1}$ :

$$
\tilde{p}_{1}^{\prime \prime}-\chi^{2} \tilde{p}_{1}=0
$$

where

$$
\chi=\sqrt{\alpha^{2}+\beta^{2}} .
$$

The appropriate solution that decays in the far-field is $\tilde{p}_{1}=\tilde{P}_{1} e^{-\chi \bar{Y}}$ where $\tilde{P}_{1}$ is a constant to be determined. Matching with the main deck pressure and the wall normal velocity, we require

$$
\tilde{p}_{1}=p_{11}, \quad \tilde{p}_{1}^{\prime}=-\alpha^{2} A \text { on } \bar{Y}=0 .
$$

The first of these conditions determines the constant of proportionality as $\tilde{P}_{1}=p_{11}$, while the second fixes the pressure displacement relation,

$$
\chi p_{11}=\alpha^{2} A .
$$

This expression describes the crucial upper deck/lower deck interplay and provides a closure to the lower deck problem which we consider next.

\subsubsection{The lower deck expansions}

We introduce the lower deck wall normal variable $Y=\varepsilon^{-1} y$ and, motivated by the scaling analysis, our flow-field takes the form

$$
\begin{aligned}
u & =\varepsilon Y+\sigma\left[u_{1}(Y) E+c . c .\right]+\cdots \\
v & =\varepsilon^{4} v_{b}+\sigma \varepsilon^{2}\left[v_{1}(Y) E+c . c .\right]+\cdots, \\
w & =\sigma\left[w_{1}(Y) E+c . c .\right]+\cdots \\
p & =p_{0}+\sigma \varepsilon\left[p_{1} E+c . c .\right]+\cdots
\end{aligned}
$$



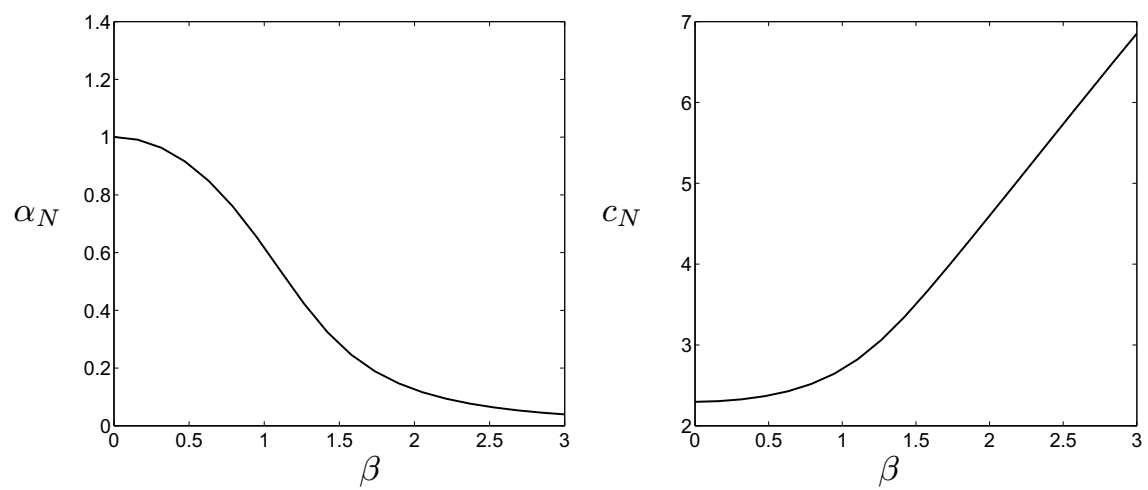

Fig. 2 Neutral values $\left(\alpha_{N}, c_{N}\right)$ for scaled wavenumber and phasespeed, as functions of scaled spanwise wavenumber $\beta$ from the solution of the lower branch eigenrelation (2.24).

The usual substitution procedure leads to the following governing equations for the perturbations in the lower deck:

$$
\mathrm{i} \alpha u_{1}+v_{1}^{\prime}+\mathrm{i} \beta w_{1}=0, \quad \mathrm{i} \alpha(Y-c) u_{1}+v_{1}=-\mathrm{i} \alpha p_{1}+u_{1}^{\prime \prime}, \quad \mathrm{i} \alpha(Y-c) w_{1}=-\mathrm{i} \beta p_{1}+w_{1}^{\prime \prime},
$$

where $p_{1}$ is a constant, and matching with the main deck we find $p_{1}=p_{11}$. These equations are to be solved subject to no slip boundary conditions on the plate

$$
u_{1}=v_{1}=w_{1}=0 \text { on } Y=0,
$$

and far field matching conditions with the main deck,

$$
u_{1} \rightarrow A, w_{1} \rightarrow 0 \text { as } Y \rightarrow \infty .
$$

The system of equations (2.19)-(2.21) can be manipulated to yield the following relationship between the pressure and the displacement:

$$
-\chi^{2} p_{11}=\alpha_{0}^{5 / 3} \mathcal{G}_{0} A .
$$

In the above we have defined

$$
\xi_{0}=-\mathrm{i}^{1 / 3} \alpha^{1 / 3} c, \quad \kappa_{0}=\int_{\xi_{0}}^{\infty} \operatorname{Ai}(s) d s, \quad \mathcal{G}_{0}=\mathrm{i}^{5 / 3} \frac{\operatorname{Ai}^{\prime}\left(\xi_{0}\right)}{\kappa_{0}},
$$

where $\mathrm{Ai}$ is the usual Airy function.

\subsubsection{The linear dispersion relation}

Combining the two equations $(2.17),(2.22)$ relating the pressure and the displacement, leads us to the linear dispersion relation

$$
\mathcal{D}(\alpha, c, \beta) \equiv \mathcal{G}_{0}+\alpha^{1 / 3} \chi=0,
$$




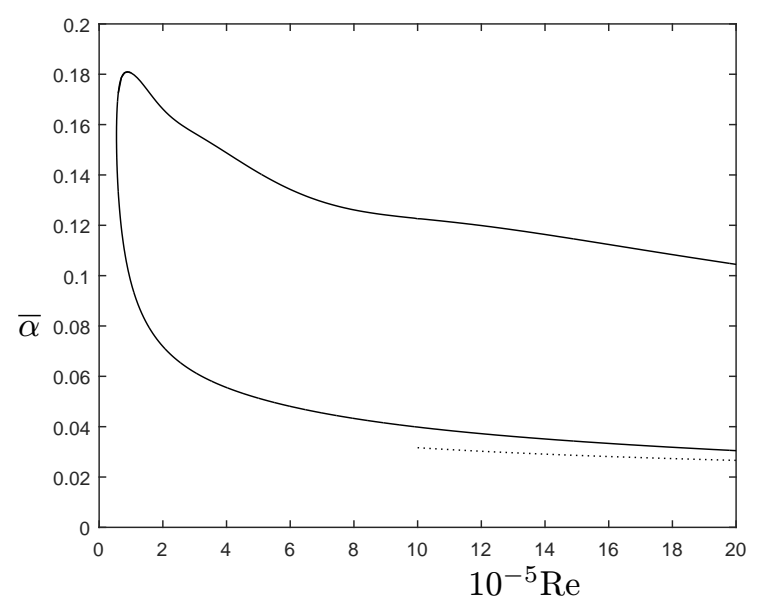

Fig. 3 Solid curve: linear neutral curve for ASBL at spanwise wavenumber $\bar{\beta}=\operatorname{Re}^{-1 / 4}$, (i.e. $\beta=1)$. Dotted curve: the lower branch asymptote derived in $(2.25)$.

with $\chi$ defined in (2.15). Expression (2.24) is a complex-valued equation which for a given scaled spanwise number $\beta$, can be solved for the two real neutral values $(\alpha, c)=\left(\alpha_{N}, c_{N}\right)$, say. Fig. 2 shows plots of the neutral streamwise wavenumber $\alpha_{N}(\beta)$ and the wavespeed $c_{N}(\beta)$ as the spanwise wavenumber varies. In the remainder of the paper we will concentrate on $\beta=1$. In this case we can solve $\mathcal{D}\left(\alpha_{N}, c_{N}, 1\right)=0$ to find $\left(\alpha_{N}, c_{N}\right)=(0.617,2.698)$ to three decimal places. Thus, as $\operatorname{Re} \rightarrow \infty$, (and in view of the scalings (2.8)), the lower branch neutral curve is described by

$$
\bar{\alpha} \sim 0.617 \mathrm{Re}^{-1 / 4}
$$

In Fig. 3 we show this lower branch asymptote and the corresponding finite Reynolds number neutral curve calculated from the numerical solution of the modified Orr-Sommerfeld equation (2.3) with $\bar{\beta}=\mathrm{Re}^{-1 / 4}$. One can see that the asymptotic result gives a good approximation to the lower branch provided Re is $O\left(10^{6}\right)$ or larger.

\section{A self-sustaining interaction in the asymptotic suction boundary layer}

In this section we will increase the amplitude $\sigma$ of the wave perturbation to the critical size at which nonlinear effects are no longer negligible. In fact we will find that the selfinteraction of the wave in the lower deck sets into motion a strongly nonlinear process that induces an $O(1)$ mean-flow distortion to the basic state. We will see presently that the wave interaction manifests itself through an effective spanwise wall slip velocity on the main deck problem. First we employ an order of magnitude analysis in order to derive the corresponding scalings for the nonlinear interaction, identifying a distinguished scaling for $\sigma$ in terms of Re. The wave scalings are essentially found from the linear stability arguments of section 2.2, the only difference being that the basic flow driving the motion is no longer given by the simple form (1.8), (1.9), but is now a streak flow, dependent on 
both $y$ and the spanwise coordinate $Z$. Let us consider the steady, $x$-independent part of the flow in the main deck and now suppose it to be three-dimensional so that it possesses three components $(U, V, W)$, say. The quantity $U(y, Z)$ is the streak flow referred to above, while $(V(y, Z), W(y, Z))$ is known as the roll flow. For nonlinearity to be key at leading order we require $V U_{y} \sim \varepsilon^{4} U_{y y}$, with $\varepsilon$ given in (2.5), and thus the normal roll component $V=O\left(\varepsilon^{4}\right)$. Over the slow spanwise scale $z \sim O\left(\varepsilon^{-1}\right)$, in order to balance terms in the continuity equation (1.6a), we require a spanwise roll component $W=O\left(\varepsilon^{3}\right)$. Now let us consider the analogous streak/roll components in the lower deck problem, denoting these as $(\bar{u}, \bar{v}, \bar{w})$. Here the nonlinear self interaction of the wave drives the roll flow directly, so if we consider the spanwise momentum equation we find that the wave forcing terms must be in balance with the wall normal diffusive term via the equation $\left[v_{1}^{*} w_{1 z}+c . c\right] \sim \varepsilon^{4} \bar{w}_{y y}$. We therefore deduce that the spanwise roll component in the lower deck has size $\bar{w}=O\left(\varepsilon^{-1} \sigma^{2}\right)$. The wave components decay algebraically away from the wall, and it turns out (as we will see shortly) that this induces the spanwise component of the roll to grow logarithmically away from the wall, and therefore to reach a size of order $\varepsilon^{-1} \sigma^{2} \log \varepsilon^{-1}$ on exit from the lower deck. Balancing this with the spanwise component of the roll in the main deck (established above to be $O\left(\varepsilon^{3}\right)$ ) we find the critical size of the wave to be

$$
\sigma=\varepsilon^{2} \delta
$$

where for convenience we have defined the small parameter $\delta$ as

$$
\delta=2(\log \operatorname{Re})^{-1 / 2}
$$

Due to nonlinear effects we must now allow for more complicated behaviour in the spanwise direction than was present in the linear regime, and with this in mind we reformulate the travelling wave contributions as unknown functions of $y$ and $Z$ multiplied by $E_{1}$ where

$$
E_{1}=\exp [i \alpha(X-c T)]
$$

We will continue to restrict our attention to solutions which are periodic in the spanwise direction with period $2 \pi / \beta$ where $\beta$ is a prescribed scaled spanwise wavenumber.

\subsection{The VWI main deck}

In the above discussion we have motivated the nonlinear extension to the scalings, so here we will just state the full expansions within the main deck which take the form

$$
\begin{aligned}
u & =U(y, Z)+\varepsilon^{2} \delta\left[u_{11}(y, Z) E_{1}+c . c .\right]+\cdots, \\
v & =\varepsilon^{3} \delta\left[v_{11}(y, Z) E_{1}+c . c .\right]+\varepsilon^{4} V(y, Z)+\cdots, \\
w & =\varepsilon^{3} W(y, Z)+\varepsilon^{3} \delta\left[w_{11}(y, Z) E_{1}+c . c .\right]+\cdots, \\
p & =p_{0}+\varepsilon^{3} \delta\left[p_{11}(Z) E_{1}+c . c .\right]+\cdots,
\end{aligned}
$$

with $\varepsilon, \delta$ given in terms of Re by $(2.5),(3.2)$. Substituting these expansions into (1.6) we find the governing equations for the roll/streak flow are

$$
V_{y}+W_{Z}=0, \quad V U_{y}+W U_{Z}=U_{y y}, \quad V W_{y}+W W_{Z}=W_{y y} .
$$


Note that, to this order of magnitude, there is no explicit wave forcing here. There is in fact a direct forcing on these equations but it arises via a boundary condition as we shall see shortly. The system (3.4) is to be solved subject to far field decay conditions

$$
U \rightarrow 1, W \rightarrow 0 \text { as } y \rightarrow \infty
$$

no slip boundary conditions on the streak $U$, and as discussed already a spanwise slip velocity for the roll

$$
U=0, \quad V=-1, W=q(Z) \text { on } y=0,
$$

with the forcing function $q(Z)$ still to be determined from a detailed analysis of the lower deck equations. Again, substituting (3.3) into (1.6), but this time considering the terms proportional to $E_{1}$, we find

$$
\mathrm{i} \alpha u_{11}+v_{11 y}=0, \quad \mathrm{i} \alpha U(y, Z) u_{11}+v_{11} U_{y}=0, \quad \mathrm{i} \alpha U(y, Z) w_{11}+p_{11 Z}=0,
$$

which is the strongly nonlinear analogue of equations $(2.10)$, where the streak profile $U(y, Z)$ is now one of the unknowns of the problem and is expected to be qualitatively different from the basic state $u_{b}(y)$. In an analogous manner to the linear case, the solution of this system can be written in the form

$$
u_{11}=A(Z) U_{y}(y, Z), \quad v_{11}=-\mathrm{i} \alpha A(Z) U(y, Z), w_{11}=-\frac{1}{\mathrm{i} \alpha U(y, Z)} p_{11}^{\prime}(Z),
$$

where now the displacement $A=A(Z)$ is an unknown function of the spanwise coordinate. Similar to the linear case we see that $u_{11}=\lambda A$ on $y=0$, where

$$
\lambda(Z)=U_{y}(y=0, Z)
$$

is no longer simply equal to unity, but is a function of the spanwise coordinate, and determined as part of the overall solution.

\subsection{The VWI upper deck}

In the upper deck we only need to consider the wave perturbations in order to close the system. The analysis is similar to the linear case, with the inclusion of the more general spanwise dependence. The flow variables expand as

$$
\begin{aligned}
u & =1+\varepsilon^{3} \delta\left[\tilde{u}_{1}(\bar{Y}, Z) E_{1}+c . c .\right]+\cdots \\
v & =\varepsilon^{3} \delta\left[\tilde{v}_{1}(\bar{Y}, Z) E_{1}+c . c .\right]+\cdots \\
w & =\varepsilon^{3} \delta\left[\tilde{w}_{1}(\bar{Y}, Z) E_{1}+c . c .\right]+\cdots \\
p & =p_{0}+\varepsilon^{3} \delta\left[\tilde{p}_{1}(\bar{Y}, Z) E_{1}+\text { c.c. }\right]+\cdots,
\end{aligned}
$$

with $\bar{Y}=\varepsilon y$ as before. Note that the suction velocity, being of $O\left(\varepsilon^{4}\right)$, is a higher order effect here. Substituting these expansions into the Navier-Stokes equations, we find the wave components satisfy the linearised Euler equations

$$
\begin{aligned}
\mathrm{i} \alpha \tilde{u}_{1}+\tilde{v}_{1 \bar{Y}}+\tilde{w}_{1 Z} & =0, \\
\mathrm{i} \alpha \tilde{u}_{1}+\mathrm{i} \alpha \tilde{p}_{1} & =0, \\
\mathrm{i} \alpha \tilde{v}_{1}+\tilde{p}_{1 \bar{Y}} & =0, \\
\mathrm{i} \alpha \tilde{w}_{1}+\tilde{p}_{1 Z} & =0 .
\end{aligned}
$$


Comparing this to the linear case given by equations (2.13), we see that the only difference is the more complicated spanwise dependence here caused by nonlinear effects elsewhere in the flow-field. Eliminating the velocity variables we find the equation for the wave component of the pressure in the upper deck can be written in the form

$$
\tilde{p}_{1 \overline{Y Y}}+\tilde{p}_{1 Z Z}-\alpha^{2} \tilde{p}_{1}=0,
$$

with the far field condition

$$
\tilde{p}_{1} \rightarrow 0 \text { as } \bar{Y} \rightarrow \infty .
$$

Matching with the main deck we find

$$
\tilde{p}_{1}=p_{11}(Z), \quad \tilde{p}_{1 \bar{Y}}=-\alpha^{2} A(Z) \text { on } \bar{Y}=0,
$$

exactly as in (2.16) apart from the non-trivial spanwise dependence.

\subsection{The nonlinear lower deck}

Here we will expand our discussion of the lower deck with the aim of determining explicitly the spanwise slip velocity denoted by $q(Z)$ in (3.6). We have already motivated the scale of the spanwise roll component in this region to be $w \sim \varepsilon^{-1} \sigma^{2}=\varepsilon^{3} \delta^{2}$. Consideration of the continuity balance (1.6a) determines the size of the normal roll velocity as $v \sim \varepsilon^{5} \delta^{2}$. The contributions from the wave terms are exactly as in the linear case but with the amplitude $\sigma$ now given by (3.1). The nonlinear self-interaction of the wave in both the streamwise and spanwise momentum equations generates an inertial forcing of size $\varepsilon \sigma^{2}=\varepsilon^{5} \delta^{2}$. The interaction will be self-sustained if this forcing balances the viscous decay of the roll/streak flow: this selects the magnitude of the streak and spanwise roll perturbations as $O\left(\varepsilon^{3} \delta^{2}\right)$. Thus, with $Y=\varepsilon^{-1} y$ as before, the lower deck flow variables are found to expand as

$$
\begin{aligned}
u & =\varepsilon \lambda(Z) Y+\cdots+\varepsilon^{2} \delta\left[u_{1}(Y, Z) E_{1}+c . c .\right]+\varepsilon^{3} \delta^{2} u_{S}(Y, Z)+\cdots, \\
v & =-\varepsilon^{4}+\varepsilon^{4} \delta\left[v_{1}(Y, Z) E_{1}+c . c .\right]+\varepsilon^{5} \delta^{2} v_{R}(Y, Z)+\cdots, \\
w & =\varepsilon^{2} \delta\left[w_{1}(Y, Z) E_{1}+\text { c.c. }\right]+\varepsilon^{3} \delta^{2} w_{R}(Y, Z)+\cdots \\
p & =\varepsilon^{3} \delta\left[p_{1}(Y, Z) E_{1}+\text { c.c. }\right]+\cdots
\end{aligned}
$$

where $\lambda(Z)$, given in (3.9), denotes the shear stress on the wall from the main deck. Substituting these expansions into the Navier-Stokes equations we find the equations governing both the roll/streak and wave parts of the flow. First, the equations governing the wave part are found to be

$$
\begin{aligned}
\mathrm{i} \alpha u_{1}+v_{1 Y}+w_{1 Z} & =0 \\
\mathrm{i} \alpha(\lambda(Z) Y-c) u_{1}+\lambda(Z) v_{1}+\lambda^{\prime}(Z) Y w_{1} & =-\mathrm{i} \alpha p_{1}+u_{1 Y Y}, \\
0 & =-p_{1 Y} \\
\mathrm{i} \alpha(\lambda(Z) Y-c) w_{1} & =-p_{1 Z}+w_{1 Y Y}
\end{aligned}
$$

which should be compared with the analogous linear stability equations (2.19) in which the wall shear $\lambda(Z)$ is equal to unity. As in the linear case we see that the wave pressures in the 
main and lower decks are identical and hence $p_{1}(Z) \equiv p_{11}(Z)$. The appropriate boundary conditions are no slip on the wall:

$$
u_{1}=v_{1}=w_{1}=0 \text { on } \quad Y=0
$$

and far field matching conditions:

$$
u_{1} \rightarrow \lambda(Z) A(Z), \quad w_{1} \rightarrow 0 \text { as } Y \rightarrow \infty .
$$

The system consisting of (3.16)-(3.18) can be rewritten as one equation relating the pressure and displacement:

$$
p_{11}^{\prime \prime}-\frac{\lambda^{\prime}}{\lambda} \mathcal{F}(\xi) p_{11}^{\prime}-\alpha^{2} p_{11}=(\alpha \lambda)^{5 / 3} \mathcal{G}(\xi) A
$$

(as first derived in (13); see also (14)) with

$$
\xi(Z)=-\mathrm{i}^{1 / 3} \frac{(\alpha \lambda(Z))^{1 / 3} c}{\lambda(Z)},
$$

and

$$
\kappa(\xi)=\int_{\xi}^{\infty} \operatorname{Ai}(s) d s, \quad \mathcal{F}(\xi)=\frac{3}{2}+\frac{\xi\left(\xi \kappa(\xi)+\mathrm{Ai}^{\prime}(\xi)\right)}{2 \operatorname{Ai}(\xi)}, \quad \mathcal{G}(\xi)=\mathrm{i}^{5 / 3} \frac{\operatorname{Ai}^{\prime}(\xi)}{\kappa(\xi)} .
$$

Again, this is a generalisation of the linear dispersion relation (2.22) to a spanwise-dependent basic flow and indeed (3.19) reduces back to this expression if we set $\lambda(Z) \equiv 1$ and use (3.12)-(3.14) to relate $p_{11}$ and $A$.

For calculating the nonlinear effect of the wave on the roll/streak flow in the main deck we require a knowledge of the asymptotic behaviour of the wave as we move out of the lower deck. From (3.16), (3.18) we can calculate

$$
\begin{aligned}
& u_{1} \sim \lambda A-\frac{1}{\alpha^{2}} \frac{d}{d Z}\left(\frac{1}{\lambda} \frac{d p_{1}}{d Z}\right) \frac{1}{Y}+\cdots \\
& v_{1} \sim-\mathrm{i} \alpha \lambda A Y-\frac{1}{\mathrm{i} \alpha \lambda}\left(\frac{d^{2} p_{1}}{d Z^{2}}-\alpha^{2} p_{1}+\lambda \alpha^{2} c A-\frac{2}{\lambda} \frac{d \lambda}{d Z} \frac{d p_{1}}{d Z}\right)+\cdots \\
& w_{1} \sim-\frac{1}{\mathrm{i} \alpha \lambda} \frac{d p_{1}}{d Z} \frac{1}{Y}-\frac{c}{\mathrm{i} \alpha \lambda^{2}} \frac{d p_{1}}{d Z} \frac{1}{Y^{2}}+\cdots
\end{aligned}
$$

as $Y \rightarrow \infty$. We will now see how the wave affects the roll/streak flow and sets up a self-sustaining interaction. Returning to expansion (3.15) and now considering the $x$-independent part we find that the roll/streak dynamics are described by

$$
\begin{aligned}
v_{R Y}+w_{R Z} & =0, \\
\lambda(Z) v_{R}+\lambda^{\prime}(Z) Y w_{R}+\left[v_{1} u_{1 Y}^{*}+w_{1} u_{1 Z}^{*}+\text { c.c. }\right] & =u_{S Y Y}, \\
{\left[\mathrm{i} \alpha w_{1} u_{1}^{*}+v_{1} w_{1 Y}^{*}+w_{1} w_{1 Z}^{*}+\text { c.c. }\right] } & =w_{R Y Y} .
\end{aligned}
$$

These equations must be solved subject to no slip boundary conditions on the wall,

$$
u_{S}=w_{R}=v_{R}=0 \text { on } Y=0,
$$


and far field matching conditions with the main deck which require careful consideration. If we integrate $(3.23 \mathrm{c})$ with respect to $Y$ we find

$$
w_{R Y}(Y, Z)=w_{R Y}(0, Z)+\int_{0}^{Y} F_{w}(s, Z) \mathrm{d} s,
$$

where

$$
F_{w}(Y, Z)=\left[\mathrm{i} \alpha w_{1} u_{1}^{*}+v_{1} w_{1 Y}^{*}+w_{1} w_{1 Z}^{*}+c . c .\right] .
$$

Using the asymptotic expansions (3.22) above, it is evident that $F_{w} \sim-q(Z) Y^{-2}$ as $Y \rightarrow \infty$, where

$$
q(Z)=-\frac{1}{\alpha^{2} \lambda^{2}} \frac{d}{d Z}\left(\alpha^{2}\left|p_{11}\right|^{2}+\left|\frac{d p_{11}}{d Z}\right|^{2}\right)
$$

and hence we conclude that

$$
w_{R Y} \sim C(Z)+q(Z) / Y
$$

as $Y \rightarrow \infty$, where

$$
C(Z)=w_{R Y}(0, Z)+\int_{0}^{\infty} F_{w}(s, Z) \mathrm{d} s .
$$

If the unknown function $C(Z)$ is non-zero then $w_{R}$ will grow algebraically as $Y \rightarrow \infty$, and will be too large to be accommodated within the main deck scalings. We therefore require $C(Z) \equiv 0$, which then determines the jump in spanwise shear across the lower deck. It follows that $w_{R} \sim q(Z) \log Y$ as $Y \rightarrow \infty$ and hence the boundary conditions for the main deck roll/streak problem are simply as given in (3.6), with $q(Z)$ defined in (3.25).

\subsection{Summary of the self-sustained interaction equations}

For clarity here we will list the equations governing the self-sustained process which were derived in the last three subsections and consist of three interacting parts. The first part of the interaction is the set of equations describing the roll/streak flow in the main deck:

$$
\begin{aligned}
V_{y}+W_{Z} & =0 \\
V U_{y}+W U_{Z} & =U_{y y} \\
V W_{y}+W W_{Z} & =W_{y y} .
\end{aligned}
$$

These equations show how the $(V, W)$ roll flow is nonlinear but that the streak $U$ satisfies a linear equation and is simply advected by the roll flow. The boundary conditions are

$$
U=0, \quad V=-1, \quad W=q(Z) \text { on } \quad y=0,
$$

where $q(Z)$ is defined in (3.25) and describes the effect of nonlinear wave forcing on the roll flow. Indeed it is this spanwise slip condition which allows us to compute a non-trivial solution of (3.26). In addition we have far field matching conditions with the upper deck

$$
U \rightarrow 1, \quad W \rightarrow 0 \text { as } y \rightarrow \infty
$$

The second part of the interaction is the governing equation for the wave which is in 
the form of an eigenvalue problem for the streamwise wavenumber $\alpha$ and wavespeed $c$, and takes the form

$$
\frac{d^{2} p_{11}}{d Z^{2}}-\frac{1}{\lambda} \frac{d \lambda}{d Z} \mathcal{F}(\xi(Z)) \frac{d p_{11}}{d Z}-\alpha^{2} p_{11}=(\alpha \lambda)^{5 / 3} \mathcal{G}(\xi(Z)) A(Z),
$$

with definitions of the coefficients given in (3.20), (3.21).

The third and final part of the interaction is the pressure-displacement relation which can be found from the solution of the problem

$$
\tilde{p}_{1 \overline{Y Y}}+\tilde{p}_{1 Z Z}-\alpha^{2} \tilde{p}_{1}=0,
$$

with matching conditions with the main deck

$$
\tilde{p}_{1}=p_{11}(Z), \quad \tilde{p}_{1 \bar{Y}}=-\alpha^{2} A(Z) \text { on } \bar{Y}=0,
$$

and far field decay conditions

$$
\tilde{p}_{1} \rightarrow 0 \text { as } \bar{Y} \rightarrow \infty .
$$

To close the system we specify a spanwise wavenumber $\beta$ and seek solutions periodic in the spanwise direction with period $2 \pi / \beta$. We must also give some measure of the amplitude of the wave. We do this by defining the quantity

$$
\mathcal{A}^{2}=\frac{\beta}{2 \pi} \int_{0}^{2 \pi / \beta}\left|p_{11}(Z)\right|^{2} \mathrm{~d} Z .
$$

In the absence of any wave forcing we have $\mathcal{A}=q=0$ and it can then be shown (for example using energy arguments) that the only solution to (3.26)-(3.28) for the roll/streak flow is undisturbed ASBL, i.e. $(U, V, W)=\left(u_{b}(y),-1,0\right)$. In section 4 we will compute weakly nonlinear states where we assume $\mathcal{A} \ll 1$, and these states can be described analytically. Then in section 5 we consider strongly nonlinear states with $\mathcal{A} \sim O(1)$ : these must be computed numerically, and a suitable numerical method is outlined at the beginning of the section.

\section{Weakly nonlinear analysis of the vortex-wave interaction equations}

Although the solution of (3.26)-(3.28) at $O(1)$ values of $\mathcal{A}$ is a complicated computational problem, some analytic progress can be made with these equations by considering small but finite amplitude wave perturbations. This analysis also provides a partial check on the accuracy of the full nonlinear computations which follow in section 5. Here we assume $\mathcal{A} \sim \Delta$ where $\Delta \ll 1$ so that

$$
\left(A, p_{11}\right)=\Delta\left(A_{1}, P_{1}\right) \cos \beta Z+\cdots .
$$

Since the forcing from the wave on the main deck vortex problem is, from (3.6), (3.25), of size $\left|p_{11}\right|^{2} \sim O\left(\Delta^{2}\right)$ we find the perturbation to the basic state is also $O\left(\Delta^{2}\right)$ so, for example,

$$
U(y, Z)=u_{b}(y)+\Delta^{2} u_{2}(y) \cos 2 \beta Z+\cdots,
$$

and thus the perturbation to the shear stress on the wall takes the form

$$
\lambda(Z)=1+\Delta^{2} \lambda_{2} \cos 2 \beta Z+\cdots .
$$


Turning now to the wave pressure equation (3.29), we find that the streamwise wavenumber $\alpha$ and the wavespeed $c$ are perturbed by $O\left(\Delta^{2}\right)$ from their linear neutral values $\left(\alpha_{N}, c_{N}\right)$, and so we write

$$
(\alpha, c)=\left(\alpha_{N}, c_{N}\right)+\Delta^{2}\left(\alpha_{2}, c_{2}\right)+\cdots .
$$

The correction to $A(Z)$ is also $O\left(\Delta^{2}\right)$ so we can conclude that $A$ expands as

$$
A(Z)=\Delta A_{1} \cos \beta Z+\Delta^{3}\left[A_{3}^{1} \cos \beta Z+A_{3}^{3} \cos 3 \beta Z\right]+\cdots,
$$

where the higher harmonics are generated from the interaction of the wall shear stress and wave pressure via the second term on the left-hand-side of (3.29). From the leading order contributions to this equation and the $p_{11}-A$ relation (3.30)-(3.32) we find, as expected, the linear dispersion relation $(2.24)$ is satisfied by $\left(\alpha_{N}, c_{N}\right)$. Continuing to $O\left(\Delta^{2}\right)$ in the streak equation (3.26b) we can calculate the correction term $u_{2}(y)$ in (4.1), from which $\lambda_{2}$ in (4.2) follows. At $O\left(\Delta^{3}\right)$ in the wave equation (3.29) we then find a solvability condition which describes the bifurcation of weakly nonlinear states from the linear neutral point. This condition can be written in the form

$$
\nu_{1} \alpha_{2}+\nu_{2} c_{2}+\nu_{3}\left|P_{1}\right|^{2}=0
$$

where the $\nu_{j}$ are complex-valued quantities that can be computed as functions of $\beta$. There is a degree of freedom in the choice of $\Delta$, so we can set $\left|P_{1}\right|=1$ without loss of generality. This leaves a single complex-valued equation for two real unknown quantities, $\alpha_{2}$ and $c_{2}$. Fig. 4 shows numerical solutions for these quantities as $\beta$ is varied. At first sight from Figs $4(\mathrm{a}, \mathrm{b})$ it appears that the wavenumber/wavespeed corrections increase/decrease monotonically with $\beta$. However closer inspection (Figs $4(\mathrm{c}, \mathrm{d})$ ) reveals that the bifurcation changes criticality twice within the range of spanwise wavenumbers considered here. On these two occasions $\alpha_{2}$ passes through zero and thus the weakly nonlinear analysis is valid even around these critical spanwise wavenumbers. For small $\beta$ the bifurcation is supercritical, then it changes to subcritical, and back to supercritical where it appears to remain as $\beta$ is increased further. All of the computations to be described in section 5 are for unit spanwise wavenumber, so we expect a supercritical bifurcation with both $\alpha_{2}$ and $c_{2}$ greater than zero. In fact for $\beta=1$, we find that to three decimal places,

$$
\left(\alpha_{2}, c_{2}\right)=(1.629,0.723)
$$

so, noting that $\mathcal{A}^{2}=\Delta^{2} / 2$ at leading order from (3.33) and recalling the linear neutral values from section 2 , we find that the bifurcation is described up to $\left(\mathcal{A}^{2}\right)$ by

$$
\alpha \simeq 0.617+3.358 \mathcal{A}^{2}, \quad c \simeq 2.698+1.446 \mathcal{A}^{2}
$$

for small $\mathcal{A}$, and we will compare this result with numerical solutions of the full interaction equations in section 5.2 .

\section{Strongly nonlinear computations of the VWI system}

\subsection{Numerical method}

In this section we will consider the numerical solution of the vortex-wave interaction problem set out in (3.25)-(3.33) which describes self-sustaining solutions in the asymptotic boundary 

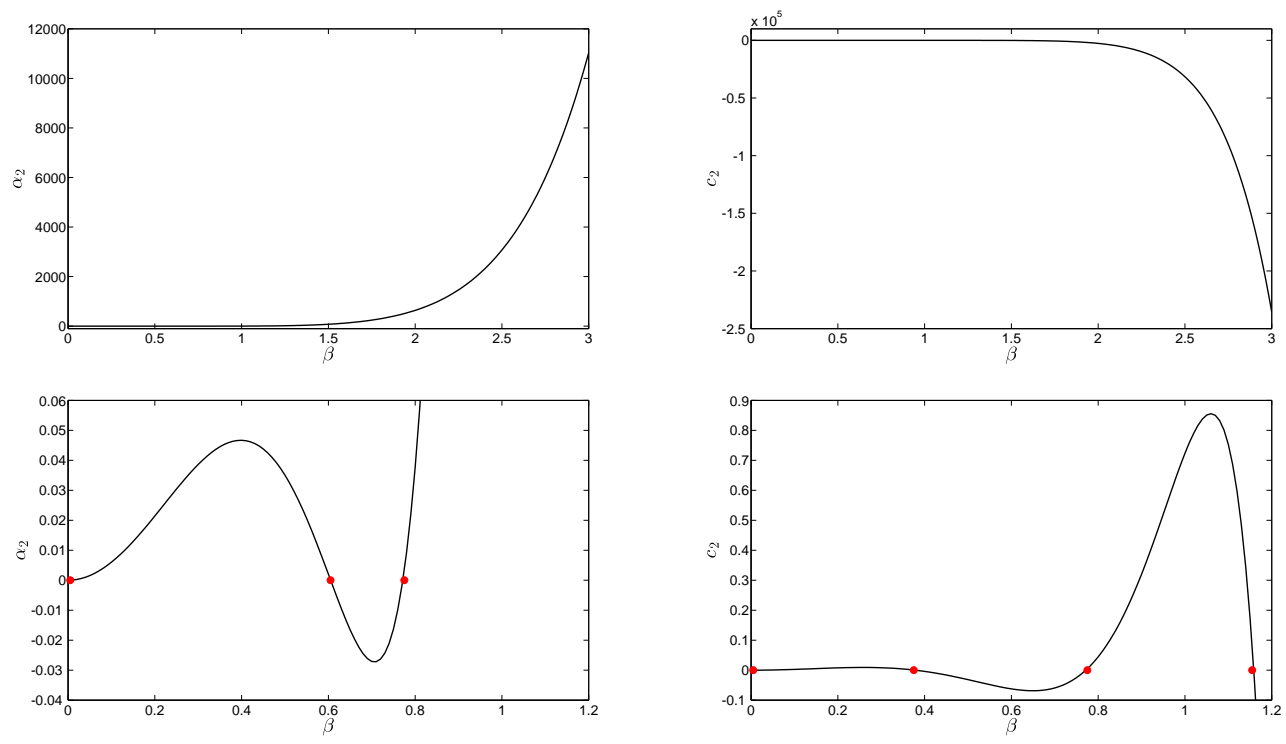

Fig. 4 The bifurcation parameters $\left(\alpha_{2}, c_{2}\right)$ from the weakly nonlinear analysis of section 4 . Fig. $4 \mathrm{c}$ shows $\alpha_{2}(\beta)$ zoomed in on $\beta \in(0,1.2)$, while Fig. $4 \mathrm{~d}$ is a zoomed-in version of $c_{2}(\beta)$ over the same range. The dots denote points where $\alpha_{2}=0$ in (c) or $c_{2}=0$ in (d).

layer at high Reynolds number. The method employed here is similar to that used by Dempsey et al. (14) to solve a related interaction problem in plane Poiseuille flow. However in that case the effect of the wave manifested itself as a forcing term in the roll/streak equations and there was a simple explicit relationship between $p_{11}$ and $A$ : instead we now have the additional complication of needing to solve the upper deck pressure-displacement system (3.30)-(3.32). This adds an extra level of complexity to the problem.

Since both the main and upper deck problems are specified over a semi-infinite range in the normal variable, we opt to transform onto a bounded region by using the exponential maps

$$
\widehat{y}=1-2 \mathrm{e}^{-y}, \quad \widehat{Y}=1-2 \mathrm{e}^{-\bar{Y}}
$$

The transformed domains for both problems is $[-1,1] \times[0,2 \pi / \beta]$. We can now apply expansions in Chebyshev polynomials in the $\widehat{y}$ and $\widehat{Y}$ directions, and exploit symmetries in the spanwise direction, to decompose the solutions into Fourier sine and cosine series. The 
unknown variables can then be written in the form

$$
\begin{aligned}
(U, V) & =\sum_{n, k=1}\left(U_{n, k}, V_{n, k}\right) T_{n-1}(\widehat{y}) \cos 2(k-1) \beta Z, \\
W & =\sum_{n, k=1} W_{n, k} T_{n-1}(\widehat{y}) \sin 2 k \beta Z, \\
\left(A, p_{11}\right) & =\sum_{k=1}\left(A_{k}, p_{11 k}\right) \cos (2 k-1) \beta Z, \\
\tilde{p}_{1} & =\sum_{n, k=1} \tilde{P}_{n, k} T_{n-1}(\widehat{Y}) \cos (2 k-1) \beta Z .
\end{aligned}
$$

The series expansions are terminated at $n=N$ and $k=K$ which are chosen to be large enough that sufficient decay in the spectral coefficients is observed. In order to resolve the spanwise variation observed in the nonlinear states it was necessary to take a value of $K$ in the range $100-250$. The structure of the solutions in the wall normal direction is more slowly-varying and a value of $N$ in the range $20-25$ proved sufficient. Expansions (5.1) are substituted into (3.26)-(3.32) and we then apply a collocation method in the wall normal direction and a Galerkin approach in the spanwise direction. Nonlinear terms are evaluated using the pseudospectral method: the product is calculated in physical space and then transformed back into Fourier space. Due to the nonlinear nature of the VWI system we must apply a de-aliasing strategy, the simplest of which is zero padding. This involves computing nonlinear products over say $\bar{K}>K$ points in physical space before computing the $K$ spectral coefficients of the product in Fourier space.

Once the collocation and Galerkin methods have been applied we are left with a residual vector which is a highly nonlinear function of the spectral coefficients. To solve this system of equations we apply a quasi-Newton method, calculating the Jacobian matrix by taking finite differences of the residual vector with respect to each spectral coefficient and the unknown streamwise wavenumber and phasespeed. This is computationally expensive but since columns of the Jacobian are calculated independently this process can be parallelised. The convergence properties of this method allow us to compute highly nonlinear states.

\subsection{Results for unit scaled spanwise wavenumber}

In this section we present some solutions to the VWI system which were computed using the method described in the previous subsection. We will only consider solutions at unit scaled spanwise wavenumber $\beta=1$, although the expectation is that the states will be similar for other spanwise wavenumbers as was found in plane Poiseuille flow (14). We will start by computing small amplitude states which can be compared with the weakly nonlinear analysis of section 4 . Then we will move on to consider situations where strongly nonlinear effects come into play.

First consider Fig. 5 which shows a comparison of the streamwise wavenumber and wavespeed computed for various amplitudes $\mathcal{A}$ from the full nonlinear equations with the predictions for small amplitudes from the weakly nonlinear analysis. Good agreement is observed, confirming that the numerical method is accurately capturing the small amplitude states. However once $\mathcal{A}$ is in excess of 0.1 , significant deviations between the two approaches are detected, indicating that nonlinear effects are important even at relatively small numerical values of $\mathcal{A}$. 

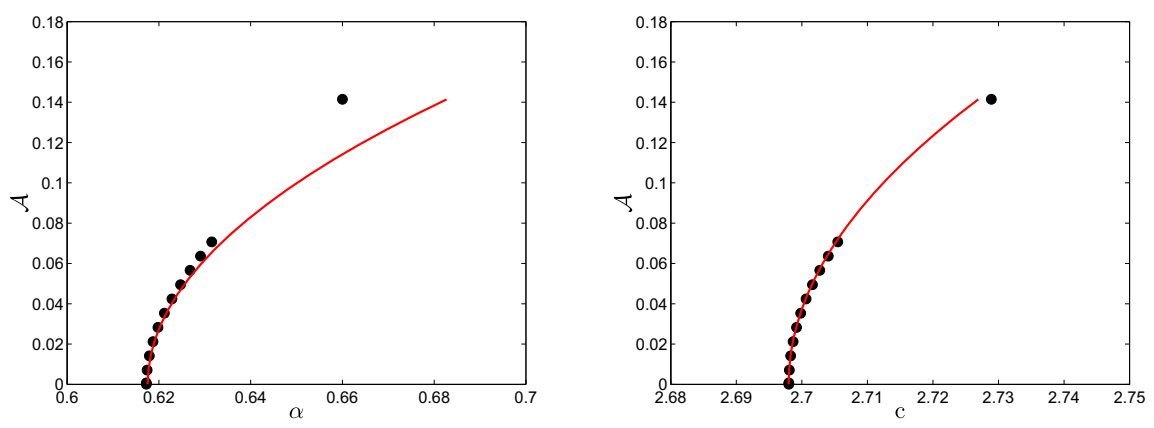

Fig. 5 Scaled wavenumber $\alpha$ and phasespeed $c$ as function of amplitude $\mathcal{A}$. The dots represent fully nonlinear computations, while the solid curves arise from the weakly nonlinear analysis.
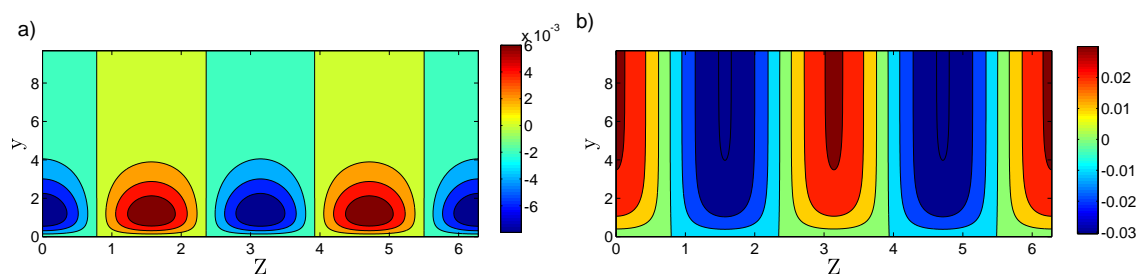

c)
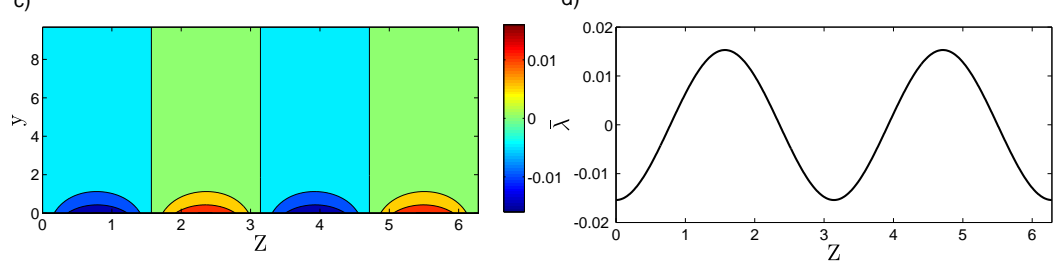

Fig. 6 Fully nonlinear computations for $\mathcal{A}^{2}=0.005$. (a) The perturbation to the streak basic state $U(y, Z)-u_{b}(y) ;(\mathrm{b}, \mathrm{c})$ the perturbation to the roll components $\left(V(y, Z)-v_{b}, W(y, Z)\right) ;(\mathrm{d})$ the perturbation to the shear stress of the basic state $\bar{\lambda}(Z)=\lambda(Z)-1$.

Next we will visualise the types of structures found in these states at various amplitudes. In each of Figs 6-8 we present contour plots in the $Z-y$ plane of the perturbation to the streak $U-u_{b}$, the perturbations to the roll components $V-v_{b}$ and $W$, and also a plot of the perturbation to the shear $\bar{\lambda}=\lambda-1$. Fig. 6 shows the state computed at an amplitude of $\mathcal{A}^{2}=0.005$, which is within the range of applicability of the weakly nonlinear analysis. As expected a regularly spaced spanwise structure is observed, consistent with the weakly nonlinear expansions (4.1), (4.2). Fig. 7 shows the situation when we increase the amplitude to $\mathcal{A}^{2}=0.02$ so that we are now out of the range of validity of the weakly nonlinear analysis. It is evident from studying the spanwise variations that nonlinear effects now play a significant role in determining the flow-field. Increasing the amplitude of the state just slightly to $\mathcal{A}^{2}=0.0242$ (Fig. 8) we see the change to the basic 

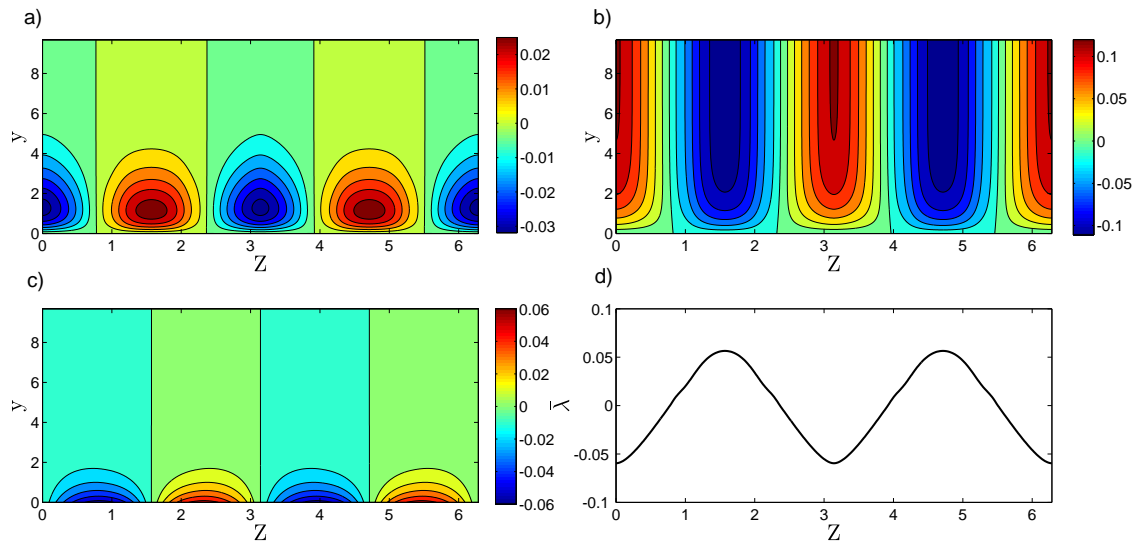

Fig. 7 Fully nonlinear computations for $\mathcal{A}^{2}=0.02$. (a) The perturbation to the streak basic state $U(y, Z)-u_{b}(y) ;(\mathrm{b}, \mathrm{c})$ the perturbation to the roll components $\left(V(y, Z)-v_{b}, W(y, Z)\right)$; (d) the perturbation to the shear stress of the basic state $\bar{\lambda}(Z)=\lambda(Z)-1$.
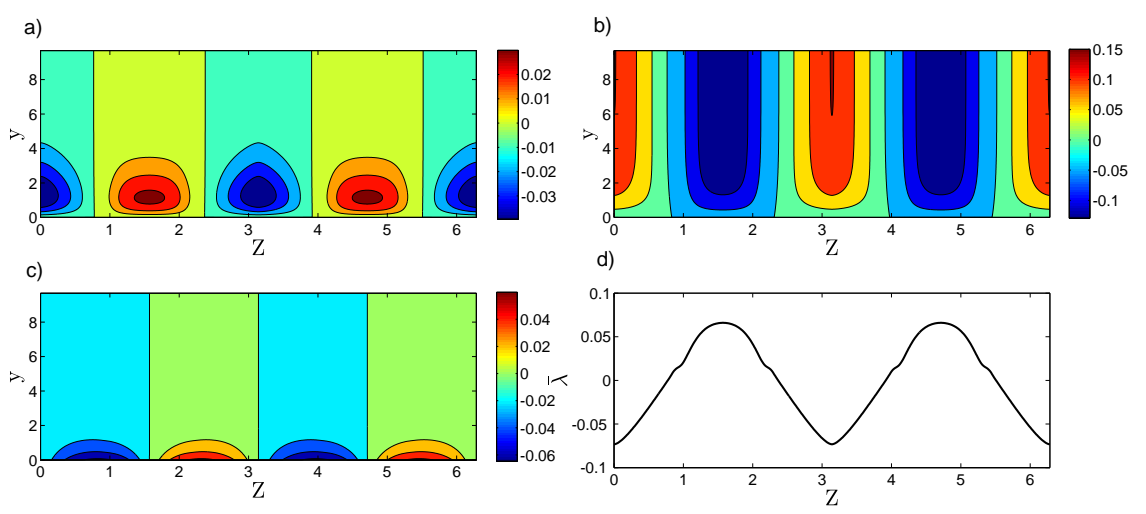

Fig. 8 Fully nonlinear computations for $\mathcal{A}^{2}=0.0242$. (a) The perturbation to the streak basic state $U(y, Z)-u_{b}(y) ;(\mathrm{b}, \mathrm{c})$ the perturbation to the roll components $\left(V(y, Z)-v_{b}, W(y, Z)\right) ;(\mathrm{d})$ the perturbation to the shear stress of the basic state $\bar{\lambda}(Z)=\lambda(Z)-1$.

state has increased significantly and in fact the magnitude of the perturbation to the streak has increased by around $50 \%$. We now start to observe rapid variations in the streak profile and wall shear stress at specific spanwise locations: in particular the shear stress profiles are becoming very sharp at spanwise locations near to $Z=0, \pi, 2 \pi$ and the roll flow appears to be focussing around these locations. If we attempt to increase the amplitude further it becomes increasingly difficult to obtain converged solutions. A solution was obtained at $\mathcal{A}^{2}=0.0451$ (and the streak profile is shown in Fig. 9) but analysis of its Fourier and Chebyshev coefficients indicated that it was under-resolved. Moreover the decay of the 


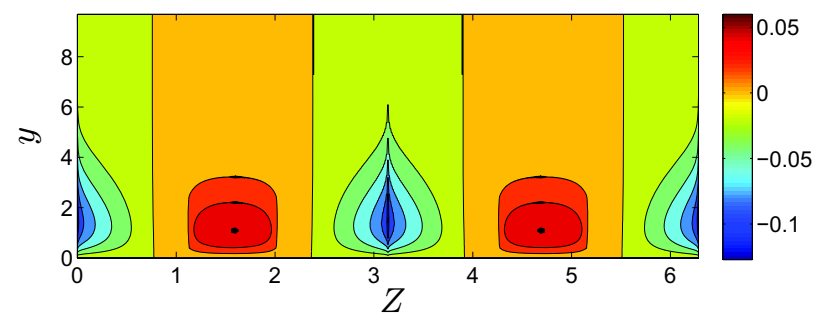

Fig. 9 Under-resolved result for $\mathcal{A}^{2}=0.0451$ showing the perturbation to the streak basic state $U(y, Z)-u_{b}(y)$.

spectral coefficients in this case is so slow that it would be impossible, given our current computing resources, to be able to repeat the computation with sufficient Fourier modes to capture accurately the flow-field at this amplitude. Nevertheless it is instructive to see how the streak profile is clearly developing a cusp at $Z=n \pi$ for $n=0,1,2$, while the solution remains smooth at other spanwise locations. The numerical evidence points to the fact that the solution appears to be losing regularity at specific spanwise locations as the amplitude is increased. A spanwise-local analytic analysis in the next section shows this to indeed be the case.

\section{Local analysis of the VWI equations}

We have seen that a feature of the numerical solutions presented in the previous section is that a localisation process begins to occur as the amplitude is increased with an apparent loss of regularity of the solution around specific spanwise locations. In this section we will show that this behaviour can be explained by examining analytically the local behaviour of solutions to the roll/streak equations (3.26) around spanwise locations $\left(Z_{0}\right.$ say) where the wave forcing is zero i.e. $q\left(Z_{0}\right)=0$. It can be seen from the $\pi$-periodic expansion for $W$ in (5.1d) that it is sufficient to consider the two points $Z_{0}=\pi / 2$ and $Z_{0}=\pi$. The numerical results of section 5.2 indicate that $q^{\prime}(\pi / 2)>0$ and $q^{\prime}(\pi)<0$. The signs of these derivatives will be shown to play a crucial role in determining the regularity of the solution and we let $q_{0}=q^{\prime}\left(Z_{0}\right)$ denote this quantity. Although it is not possible to write down $q_{0}(\mathcal{A})$ explicitly, numerical computations show that $\left|q_{0}\right|$ increases as the amplitude $\mathcal{A}$ of the state is increased and thus $q_{0}$ can be regarded as a useful measure of the nonlinearity of the solution.

From the velocity expansions given in (5.1) it is evident that in the vicinity of $Z_{0}$ we have $W \propto\left(Z-Z_{0}\right)$, while $(U, V)$ remain $O(1)$. Thus locally the roll/streak components expand as

$$
\begin{aligned}
q & =\left(Z-Z_{0}\right) q_{0}+\left(Z-Z_{0}\right)^{3} q_{2}+\cdots \\
(U, V) & =\left(U_{0}(y), V_{0}(y)\right)+\left(Z-Z_{0}\right)^{2}\left(U_{2}(y), V_{2}(y)\right)+\cdots \\
W & =\left(Z-Z_{0}\right) W_{0}(y)+\left(Z-Z_{0}\right)^{3} W_{2}(y)+\cdots
\end{aligned}
$$




\subsection{Leading order solution and numerical validation}

Substituting expansions (6.1) into the roll/streak problem (3.26) we find that the leading order local solutions satisfy the nonlinear balances

$$
V_{0}^{\prime}+W_{0}=0, \quad V_{0} U_{0}^{\prime}=U_{0}^{\prime \prime}, \quad V_{0} W_{0}^{\prime}+W_{0}^{2}=W_{0}^{\prime \prime},
$$

with far field boundary conditions

$$
U_{0} \rightarrow 1, \quad W_{0} \rightarrow 0 \quad \text { as } \quad y \rightarrow \infty
$$

and no slip and forcing conditions on the wall

$$
U_{0}=0, \quad V_{0}=-1, \quad W_{0}=q_{0} \quad \text { on } \quad y=0 .
$$

Perhaps surprisingly, these nonlinear equations have an exact solution given by

$$
\begin{aligned}
U_{0} & =\frac{1-\exp \left[\left(q_{0} / B^{2}\right)\left(1-\mathrm{e}^{-B y}\right)\right]}{1-\exp \left(q_{0} / B^{2}\right)}, \\
V_{0} & =-1-\frac{q_{0}}{B}\left(1-\mathrm{e}^{-B y}\right), \\
W_{0} & =q_{0} \mathrm{e}^{-B y}
\end{aligned}
$$

where $B$ is a positive constant (to ensure far-field decay) which satisfies

$$
B^{2}-B-q_{0}=0 \text {. }
$$

The solutions to this equation for $B\left(q_{0}\right)$ are

$$
B_{ \pm}=\frac{1 \pm \sqrt{1+4 q_{0}}}{2}
$$

and since we require $B$ to be positive there are two possible solutions for $-1 / 4<q_{0}<0$, one solution for $q_{0} \geqslant 0$ and $q_{0}=-1 / 4$ and no solutions for $q_{0}<-1 / 4$. From this we see that at the local point where $q_{0}>0(Z=\pi / 2)$ we have $B=B_{+}>1$. At this point the nonlinear solution continues to exist irrespective of the largeness of $q_{0}$. However at the local point where $-1 / 4<q_{0}<0(Z=\pi)$ we have two admissible solutions: $B_{-} \in(0,1 / 2)$ and $B_{+} \in(1 / 2,1)$. The correct choice for $B$ is determined by examining the solutions to the full interaction equations that were computed in section 5.2: at the local point with $-1 / 4<q_{0}<0$ we observe the decay given by $B_{+}$i.e. the more rapid decay of the two possibilities.

We can see that this particular local nonlinear solution, being valid only provided $\left|q_{0}\right|<$ $1 / 4$, places an upper bound on the wave amplitude for which self-sustained solutions of the global VWI system are possible and begins to provide some explanation for why it becomes increasingly difficult to compute solutions at increasing amplitude. Further insight into the nature of the breakdown can be obtained by investigating the equations governing the correction to the local solution.

Before we do this we provide some numerical evidence to support the fact that the exact solution (6.2) does accurately describe the solution of the full VWI system close to the points 

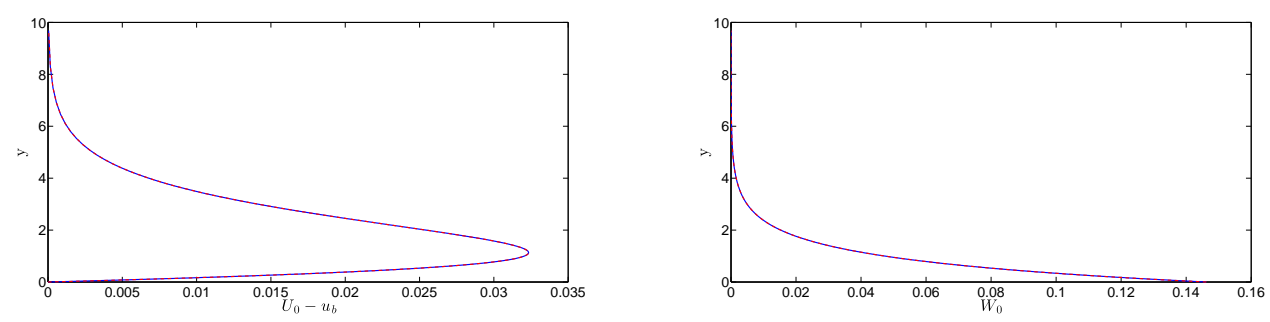

Fig. 10 Comparison of numerical results (blue solid curve) with leading-order local solution (6.2) (red dashed curve) at $Z=\pi / 2$ when $\mathcal{A}^{2}=0.0242$.
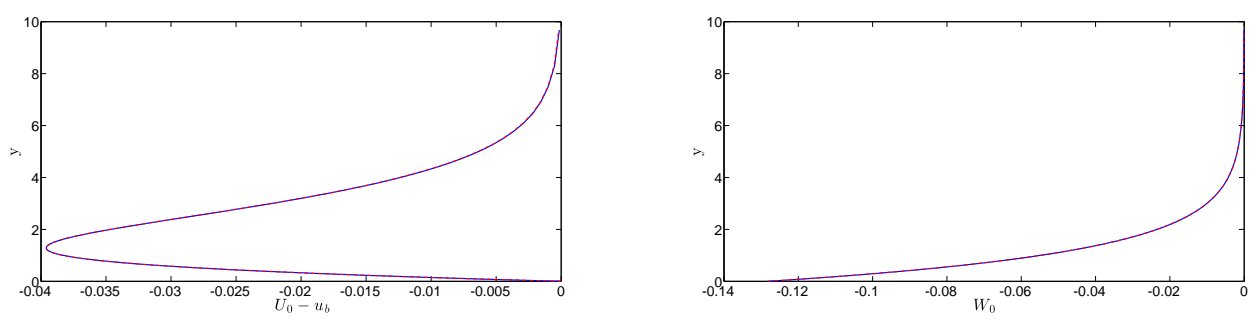

Fig. 11 Comparison of numerical results (blue solid curve) with leading-order local solution (6.2) (red dashed curve) at $Z=\pi$ when $\mathcal{A}^{2}=0.0242$.

$Z=\pi / 2$ and $Z=\pi$. We consider the solution at $\mathcal{A}^{2}=0.0242$ for which the full flow-field was plotted in Fig. 8. First let us consider the comparison at $Z=\pi / 2$. This is shown in Fig. 10 where we compare the streak perturbation and the spanwise roll component between the full solution and the analytic local form. The full numerical results give a positive value of $q_{0} \simeq 0.1462$. In this case, from (6.3), the only admissible value of $B$ is $B_{+} \simeq 1.1295$. In Fig. 11(a,b) we show the corresponding comparisons at $Z=\pi$ where it is found from the full numerical solution that $q_{0} \simeq-0.1282$. This implies, from (6.3), two possible values for $B$, with the solution to the full interaction equations corresponding to $B_{+} \simeq 0.8490$. At both local points excellent agreement between (6.2) and the full numerical solution is observed: in fact at this amplitude the results are almost completely indistinguishable. Similarly good agreement was found at other values of $\mathcal{A}$ giving us confidence that the full solution is indeed described by the simple analytical form (6.2) near these particular spanwise points.

\subsection{Higher order analysis}

Returning to the local expansion (6.1) and substituting into (3.26) we find that the roll correction $\left(V_{2}, W_{2}\right)$ satisfies

$$
V_{2}^{\prime}+3 W_{2}=0, \quad V_{0} W_{2}^{\prime}+V_{2} W_{0}^{\prime}+4 W_{0} W_{2}=W_{2}^{\prime \prime}
$$


After elimination of $W_{2}$ and use of (6.2) for the zeroth order contribution $\left(V_{0}, W_{0}\right)$ we find

$$
V_{2}^{\prime \prime \prime}+\left(B+(1-B) \mathrm{e}^{-B y}\right) V_{2}^{\prime \prime}+4 B(1-B) \mathrm{e}^{-B y} V_{2}^{\prime}+3 B^{2}(1-B) \mathrm{e}^{-B y} V_{2}=0 .
$$

The appropriate boundary conditions are

$$
V_{2}(0)=0, V_{2}^{\prime}(0)=-3 q_{2}, \quad V_{2}^{\prime}(\infty)=0 .
$$

Again an analytic solution is possible, and takes the form

$$
V_{2}=\frac{3 q_{2} B^{-1}}{\mu^{3}-9 \mu^{2}+18 \mu-6}\left(\frac{1}{2} \mu^{3} \mathrm{e}^{-3 B y}-9 \mu^{2} \mathrm{e}^{-2 B y}-\left(\frac{1}{2} \mu^{3}-9 \mu^{2}+6+18 \mu B y\right) \mathrm{e}^{-B y}+6\right),
$$

with

$$
\mu=\frac{1}{B}-1
$$

We therefore see that there is a singularity in the solution for $V_{2}$ (together with a corresponding singularity in $U_{1}$ and $W_{1}$ ) when the denominator in (6.5) is zero. This gives three real values of $B$ at which the local expansion breaks down. Recalling that $B \in(1 / 2,1)$, the relevant root is

$$
B_{\text {crit }} \simeq 0.706 \text {. }
$$

This value of $B$ corresponds to a global nonlinear state with a value of $\mathcal{A}^{2}$ that is larger than that shown in Fig. 8 (where the streak is beginning to sharpen at $Z_{0}=\pi$ ) but less than that depicted in Fig. 9 where there has been a clear loss of regularity in the flow-field near the local point. Hence we can see that the results of the local analysis clearly explain the steepening phenomenon observed in the full simulations. This loss of regularity does not occur suddenly but in fact is present in the solution even at vanishingly small amplitude and is caused by the existence of an irregular local eigensolution as we shall now show.

Returning to our local solution expansion, we now represent the roll contribution in the form

$$
V=V_{0}(y)+\cdots+\left(Z-Z_{0}\right)^{\theta} V_{\theta}+\cdots, \quad W=\left(Z-Z_{0}\right)\left(W_{0}+\cdots+\left(Z-Z_{0}\right)^{\theta} W_{\theta}+\cdots\right),
$$

where the first sets of dots represent terms that are regular in $\left(Z-Z_{0}\right)$ and the index $\theta$ is unknown and non-integral. After substitution into (3.26) and elimination of $W_{\theta}$, we find that $V_{\theta}$ satisfies

$$
V_{\theta}^{\prime \prime \prime}+\left(B+(1-B) \mathrm{e}^{-B y}\right) V_{\theta}^{\prime \prime}+(\theta+2) B(1-B) \mathrm{e}^{-B y} V_{\theta}^{\prime}+(\theta+1) B^{2}(1-B) \mathrm{e}^{-B y} V_{\theta}=0,
$$

which reduces to $(6.4)$ when $\theta=2$. If we assume that the spanwise slip velocity $q(Z)$ remains regular, then the boundary conditions on (6.7) are

$$
V_{\theta}(0)=0, V_{\theta}^{\prime}(0)=0, V_{\theta}^{\prime}(\infty)=0,
$$

The solution that satisfies the first and third boundary conditions in (6.8) above can be written as

$$
V_{\theta}=C_{\theta} \mathrm{e}^{-B y} \int_{s}^{\mu} \frac{\mathcal{L}(1+\theta, \xi)}{\xi^{2}} \mathrm{~d} \xi, s=\mu \mathrm{e}^{-B y}
$$




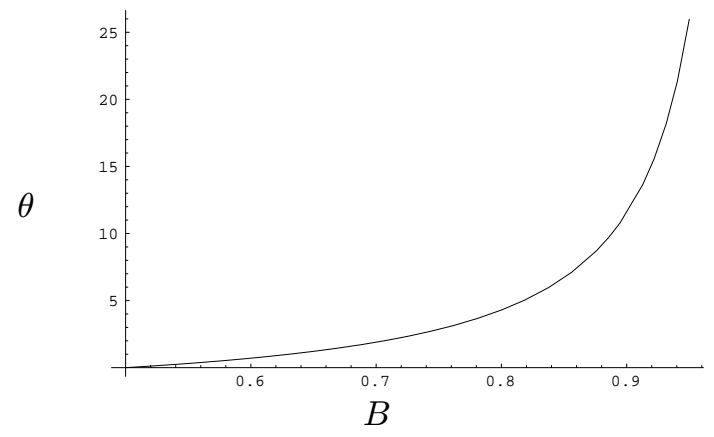

Fig. 12 Eigenvalue $\theta$ as a function of the amplitude parameter $B$ from the solution of (6.10) over the range $1 / 2<B<1$.

with $\mu$ given in (6.6) and where $\mathcal{L}(1+\theta, \xi)$ is the solution of Laguerre's equation which remains finite as $\xi \rightarrow 0$. In general the function $\mathcal{L}$ can be expressed in terms of an infinite series expansion about $\xi=0$ and reduces to the corresponding Laguerre polynomial of degree $N+1$ in the limit $\theta \rightarrow N$, with $N$ an integer: see, for example (15) for more details. Applying the second of conditions (6.8) to (6.9) we see that the eigenvalue $\theta$ satisfies

$$
\mathcal{L}(1+\theta, \mu)=0
$$

while the constant $C_{\theta}$ remains undetermined. It is this eigensolution that is responsible for the irregular behaviour evident in the roll/streak flow and shear stress plots shown in Figs. 8, 9. One can now also appreciate that the singular behaviour observed in (6.5) as $B \rightarrow B_{\text {crit }}$ is attributable to the existence of this solution: this is because the case considered above corresponds to the limit $\theta \rightarrow 2$ for which the corresponding Laguerre polynomial is the cubic in the denominator of (6.5). The index $\theta$ is plotted against $B \in[1 / 2,1]$ in Fig. 12 from the numerical solution of (6.10). It can be seen that $\theta \rightarrow 0$ as $B \rightarrow \frac{1}{2}+$. Recalling that $B=B_{+}$in (6.3), this corresponds to the limit $q_{0} \rightarrow-1 / 4$, consistent with the breakdown of the zeroth order local solution (6.2) at this value of amplitude. In addition, $\theta \propto(1-B)^{-1}$ as $B \rightarrow 1-$, which indicates that in the limit of very small amplitude $\left(q_{0} \rightarrow 0\right)$ the irregularity retreats to higher order but is always present in the solutions, even at vanishingly small amplitude. It is also worth noting that (6.10) has no solutions for $B>1$, i.e. for $q_{0}>0$. Thus the solution near to $Z_{0}=\pi / 2$ always remains regular, in agreement with the full numerical simulations.

\section{Conclusions}

In this work we have set out the large Reynolds number scales for lower branch viscous Tollmien-Schlichting waves in the asymptotic suction boundary layer based on the ideas of (11) for the Blasius boundary layer. With the important scales identified we increased the size of the wave until the correction to the roll/streak flow from the nonlinear self interaction of the wave is large enough to alter the basic state at the leading order from the ASBL profile $u_{b}(y)$ to a streaky flow $U(y, Z)$ to be determined as part of the solution. The structure was found to consist of the strongly nonlinear self-interaction of a viscous 
wave within the lower deck which generates a roll/streak flow which grows logarithmically with distance from the wall. This induces a corresponding roll/streak structure within the main deck which is large enough to alter the basic streak throughout the majority of the boundary layer. The perturbed streak suffers an instability which is propagated through the lower deck by the shear stress, sustaining the wave which drove the system away from the basic state in the first place.

Weakly nonlinear states which bifurcate from the linear neutral point were investigated analytically and good agreement with small amplitude states computed from the fully nonlinear VWI equations was found. As the value of the amplitude parameter $\mathcal{A}$ was increased the flow started to develop rapid spanwise variations near particular values of $Z$, in a similar way to computations carried out for plane Poiseuille flow (14). Once $\mathcal{A}^{2}$ exceeded 0.0242 it became very difficult to obtain a converged solution as a prohibitively large number of Fourier and Chebyshev modes were required to achieve satisfactory resolution.

A local spanwise analysis of the interaction equations was performed. An exact analytical solution of the local equations was found which is parameterised by the local gradient of the wall forcing and this solution was found to be in perfect agreement with the full numerical solution. It was found that solutions to the local equations terminate at a particular critical value of this gradient which is consistent with the difficulty in obtaining converged solutions to the global problem as the wave forcing is increased. In addition to this it was found that the local problem admits an irregular eigensolution. This solution shows how the roll/streak equations (3.26), even when subject to a regular wave forcing, gradually lose regularity as the amplitude of the forcing is increased. Indeed this eigensolution is always present, even at vanishingly small amplitude, at which stage the irregular term occurs at very high order in the local solution expansion. The existence of this irregularity at a particular value of $Z$ is responsible for the observed loss of resolution in the full numerical solution with increasing amplitude, as ever more terms in the Fourier series are required in a futile attempt to smooth out the singularity.

The loss of regularity of the VWI system appears to be due to the fact that the roll/streak equations (3.26) are only first order in $Z$ due to the thinness of the boundary layer and the relatively long spanwise lengthscale of the TS waves. The entire VWI system is not first order in $Z$ however, because of the TS pressure equation (3.29), but the ellipticity (in $Z$ ) of this equation appears to be outweighed by the parabolic nature of (3.26), resulting in the fact that a smooth periodic solution of the system cannot be guaranteed. It is likely that to resolve this issue, as speculated in (14) for plane Poiseuille flow, there are thin diffusion layers around the points $Z=0, \pi$ where the governing equations are second order in $Z$, allowing a smoothing of the outer irregular solution. The local analysis of section 6 predicts the critical value of $B$ (and hence $\mathcal{A}$ ) at which the singularity has moved up the local expansion sufficiently to render the leading order VWI framework invalid. The form of the new structure which emerges beyond this critical value will form the focus of future research.

\section{Acknowledgement}

Support from EPSRC for the first author is gratefully acknowledged. 


\section{References}

1. T. H. Hughes and W. H. Reid, On the stability of the asymptotic suction boundary-layer profile, J. Fluid Mech. 23 (1965) 715-735.

2. L. M. Hocking, Nonlinear instability of the asymptotic suction velocity profile, Q. J. Mech. Appl. Maths 28 (1975) 341-353.

3. J. H. M. Fransson and P. H. Alfredsson, On the disturbance growth in an asymptotic suction boundary-layer, J. Fluid Mech. 482 (2003) 51-90.

4. F. A. Milinazzo and P. G. Saffman, Finite-amplitude steady waves in plane viscous shear flows, J. Fluid Mech. 160 (1985) 281-295.

5. T. Khapko, T. Kreilos, P. Schlatter, Y. Duguet, B. Eckhardt and D. S. Henningson, Localized edge states in the asymptotic suction boundary layer, J. Fluid Mech. $\mathbf{7 1 7}$ (2012) R6.

6. T. Kreilos, G. Veble, T. M. Schneider and B. Eckhardt, Edge states for the turbulent transition in the asymptotic suction boundary layer, J. Fluid Mech. 726 (2013) 100122.

7. K. Deguchi and P. Hall, Free-stream coherent structures in parallel boundary-layer flows, J. Fluid Mech. 752 (2014) 602-625.

8. T. Kreilos, J. F. Gibson and T. M. Schneider, Localized travelling waves in the asymptotic suction boundary layer, Submitted to J. Fluid Mech. (2016)

9. J. M. Hamilton, J. Kim and F. Waleffe, Regeneration mechanisms of near-wall turbulence structures, J. Fluid Mech. 287 (1995) 317-348.

10. P. Hall and F. T. Smith, The nonlinear interaction of Tollmien-Schlichting waves and Taylor-Gortler vortices in curved channel flows, Proc. R. Soc. Lond. A417 (1988) $255-282$.

11. P. Hall and F. T. Smith, On strongly nonlinear vortex/wave interactions in boundarylayer transition, J. Fluid Mech. 227 (1991) 641-666.

12. F. T. Smith, On the non-parallel flow stability of the Blasius boundary layer, Proc. $R$. Soc. Lond. A366 (1979) 91-109.

13. F. T. Smith, Instability of flow through pipes of general cross-section, part 1 , Mathematika 26 (1979) 187-210.

14. L. J. Dempsey, K. Deguchi, P. Hall and A. G. Walton, Localized vortex/TollmienSchlichting wave interaction states in plane Poiseuille flow, J. Fluid Mech. 791 (2016) $97-121$.

15. E. W. Weisstein, Laguerre differential equation, From 'Mathworld - A Wolfram Web Resource'. http://mathworld.wolfram.com/LaguerreDifferentialEquation.html. 